\title{
La creatividad en el deporte: una revisión sistemática.
}

\section{Creativity in Sports: A Systematic Review}

\section{Criatividade no desporto: uma revisão sistemática}

\author{
García-Peñas, V. ${ }^{\text {; }}$ García-García, C. ${ }^{2}$; Garcés de los Fayos, E.J. ${ }^{3}$; Corbalán, J. ${ }^{4}$ \\ 1,2,3,4 Departamento de Personalidad, Evaluación y Tratamiento Psicológicos. Facultad de Psicología. \\ Universidad de Murcia.
}

\begin{abstract}
RESUMEN
El deporte, a través del recurso psicológico de la creatividad, se ha ido innovando y adaptando a los cambios en los últimos años. Esta fortaleza cognitiva y afectiva está ahora más presente tanto en deportistas como en clubs. Tras una revisión sistemática de la bibliografía aportada por diversas bases de datos en los últimos 25 años, se ha realizado un cribado manual basado en unos criterios de selección previos, donde se han obtenido 39 estudios con una temática de 'creatividad y deporte' de un total de 5.174. Pese a que 14 de los 39 trabajos presentan limitaciones que podrían afectar a la replicabilidad de sus estudios o, incluso, a la calidad de sus resultados, se puede observar un creciente interés de la comunidad científica por esta materia, dándose un aumento en las publicaciones de artículos que se han centrado en esta temática. No obstante, se concluye que la investigación publicada hasta la fecha, aunque ha abierto excelentes líneas de trabajo y posibilidades, no puede ofrecerse todavía como un conjunto de contenidos, estructura o relaciones que configure un dominio específico deportivo o motriz, dentro de los recursos de creatividad.
\end{abstract}

Palabras clave: deporte, creatividad, revisión sistemática, psicología.

\begin{abstract}
Thanks to the psychological resource of creativity, sports have innovated and adapted to changes in recent years. This cognitive and affective strength is now more present in both athletes and clubs. After a systematic review of the bibliography published over the last 25 years through various databases, a manual screening was carried out based on specific selection criteria. This search provided 39 studies addressing the theme of 'creativity and sport' out of a total of 5.174 papers. Although 14 of these 39 studies have limitations that could affect their replicability, or even the quality of their results, it is possible to observe a growing interest of the scientific community in this topic, as well as an increase in the number of articles published that have focused on this subject. Thus, it is possible to conclude that, although the research published to date has opened excellent lines of work and possibilities, it cannot yet be considered as a set of content, structure or relationships shaping a specific sports or motor domain, within the resources of creativity.
\end{abstract}

Keywords: sport, creativity, systematic review, psychology. 


\section{García-Peñas et al.}

\section{RESUMO}

O desporto, através do recurso psicológico da criatividade, tem vindo a inovar e a adaptar-se às mudanças nos últimos anos. Esta força cognitiva e afectiva está agora mais presente tanto nos atletas como nos clubes. Após uma revisão sistemática da bibliografia fornecida por várias bases de dados ao longo dos últimos 25 anos, foi efectuada uma análise manual baseada em critérios de selecção anteriores, que resultou em 39 estudos com um tema de "criatividade e desporto" de um total de 5.174. Apesar de 14 dos 39 estudos terem limitações que podem afectar a reprodutibilidade dos seus estudos, ou mesmo a qualidade dos seus resultados, observa-se um interesse crescente da comunidade científica por este tema, bem como um aumento do número de artigos publicados que incidiram sobre este tópico. Contudo, conclui-se que a investigação publicada até à data, embora tenha aberto excelentes linhas de trabalho e possibilidades, ainda não pode ser oferecida como um conjunto de conteúdos, estrutura ou relações que configurem um determinado desporto ou domínio motor, dentro dos recursos da criatividade.

Palavras chave desporto, criatividade, revisão sistemática, psicología.

\section{INTRODUCCIÓN}

La creatividad está considerada desde hace años como un recurso psicológico de gran relevancia, que puede afectar a un amplísimo campo de la actividad humana. Por su parte, el deporte genera un entorno muy favorable a la superación y la mejora de las destrezas que implica. "Los atletas saltan más alto, corren más rápido y son más fuertes que nunca. La competencia se está volviendo cada vez más desafiante, impredecible y distractora" (Richard y Runco, 2020, p.632). Aunque no toda práctica deportiva implica necesariamente objetivos competitivos, sino que también los hay de otro tipo, como expresivos, instrumentales (por ejemplo, educativos), o de espectáculo (Puig y Heinemann, 1991), en general se trata de un contexto muy favorable a la innovación, ya que suele conllevar una perseverante tarea de mejora de las habilidades que entran en juego.

En el deporte, sobre todo en el ámbito competitivo, dicha apuesta por la innovación resulta evidente en los avances en las técnicas, en las estrategias, en la promoción de la forma física, o en la alimentación. Pero igualmente resulta muy relevante en el campo de la actitud personal, en la fortaleza mental o en la gestión emocional. De ahí la relevancia, junto a un gran número de otras variables, de aquellas en cuyo estudio puede hacer aportaciones la psicología (Hackfort, y Schinke, 2020; Taylor, y Wilson, 2005; Cox, 2008). Cada vez más, el deporte es multidisciplinar, y en éste, el papel de la psicología del deporte resulta más significativo, en favor de un universo deportivo que trata al deportista en su globalidad (Garcés de los Fayos, et al., 2004; Ortín-
Montero, et al., 2013). En este contexto, algunos autores, incluso, promueven una visión de la psicología del deporte identificada casi en exclusiva con la promoción y el apoyo a la excelencia en el rendimiento (Aoyagi et al., 2012), en el que la apuesta por la innovación resulta crucial.

Un recurso clave para la innovación es la creatividad, una variable psicológica que no siempre resulta fácil operativizar dentro del deporte. A pesar de ello, Memmert (2006, 2007, 2010, 2011a, 2015) ha hecho un trabajo muy relevante sobre las posibles vías de contribución de la creatividad al mundo del deporte, definiéndola como una conducta "inusual, innovadora, de rareza estadística o incluso singularidad en las soluciones a una situación deportiva relacionada" (Memmert, 2011b, p. 373).

La creatividad, indica, está muy ligada a la toma de decisiones y a la diferencia entre el pensamiento convergente y el divergente, definida por Guilford (1967). A diferencia del pensamiento convergente, que generalmente conduce a soluciones convencionales y "correctas", el pensamiento divergente se define como el proceso de generar muchas ideas alternativas (Runco, 2014) y se desarrolla en un universo que no reconoce, de entrada, límites ni exclusiones. Esta noción se ha convertido en la clave del mecanismo que subyacen a la creatividad, es la dimensión que mejor ejemplifica la manera en que el cerebro creador actúa cognitivamente, y supone una variable susceptible de ser cuantificada. Divergencia equivale a considerar distintas perspectivas, buscar más de una respuesta, desarticular 


\section{Creatividad y deporte}

esquemas rígidos, no apoyarse en suposiciones únicas y previas, "es decir, ensayar, establecer nuevas asociaciones, seleccionar de modo no usual, establecer reestructuraciones sobre lo aparentemente insólito o inútil, lanzarse por caminos inesperados, tantear para producir algo nuevo o desconocido" (Torrance y Myers, 1976, p. 11).

Estos conceptos fueron trasladados al mundo del deporte por Klaus Roth (Memmert, 2011b), siendo utilizados principalmente para optimizar los recursos psicológicos de los deportistas. De esta forma, se pretendía extender el uso de los mismos no sólo entre profesionales de la psicología que trabajaran mano a mano con los deportistas, sino para que los propios deportistas, entrenadores, e incluso las instituciones deportivas los comprendieran y les dieran uso (Memmert, 2015). Y ello con la finalidad de ayudar a identificar su potencial, alimentar las fortalezas, disminuir limitaciones y aumentar posibilidades en la adquisición de habilidades, tanto dentro como fuera del entorno deportivo. Por esto, cada vez más existe un interés en que la investigación y la aplicación de la creatividad llegue directamente a los atletas, apoyando su desarrollo. (Santos et al, 2016). Un reflejo de esta actitud, por ejemplo, es la promoción la práctica de "Flow" (fluir), un concepto muy relacionado con la creatividad, que Cox (2008) incluye de pleno en su Manual de Psicología del Deporte.

De hecho, como señala Meier (2014), en muchas ocasiones, los entrenadores, los jugadores, los equipos deportivos enteros y hasta los propios directivos no saben bien cómo hacer para promover su interés por la innovación. Resulta evidente su búsqueda de mejoras, pero en una mayoría de ocasiones ésta se suele enfocar hacia exigir más esfuerzo o más compromiso a los deportistas en un nivel técnico. Y sin embargo, en ocasiones dicha mejora es más posible que pueda llegar como resultado de dejar de insistir en las exigencias y puede "venir como resultado de realizar cambios" (Meier, 2014, p. 1). Es en ese contexto donde tienen especial cabida variables psicológicas como la resiliencia, la motivación, la autoconfianza, la generación de ideas $\mathrm{y}$, en particular, los aspectos relacionados con la aplicación de la creatividad al deporte (Pérez Ramírez, 2002).

Así pues, durante la última década, las relaciones entre creatividad y deporte se están tomando cada vez en mayor consideración (Memmert, 2015; Santos et al.,
2016). En concreto, se estudia cómo en la tarea ejecutiva deportiva, el talento creativo podría afectar a una amplia gama de procesos, que van desde la generación de una multiplicidad de ideas que el deportista proyecta (Meier, 2014) hasta la elección de cómo realizar las tareas que han sido elegidas, o a cómo implantar el despliegue de las mismas. Incluso puede resultar clave para marcar nítidamente la diferencia entre uno y otro deportista o equipo, trayendo lo imprevisible al terreno de juego y perturbando a los oponentes (Memmert, 2011a). Así, autores como Santos et al. (2017), Hüttermann et al. (2019), o Aydin (2019a, 2019b) se han dedicado a estudiar, recientemente, aspectos más específicos de esta relación entre la innovación y su evolución en el campo del deporte.

Así pues, hay una gran cantidad de estudios que han subrayado la importancia de la creatividad en el deporte (Aydin, 2019a; Aydin, 2019b; Bowers et al., 2014; Santos et al., 2017) y hay evidencias de que la creatividad parece ser una variable que afecta a la práctica deportiva (Klatt et al., 2019; Memmert et al., 2010; Conesa-Ros, y Angosto, 2017; Marín, et al., 2012). No obstante, al realizar un acercamiento a la investigación que realizan los autores más destacados en el campo de la psicología de la creatividad, no parece habitual la inclusión del estudio de la creatividad deportiva. Así, en los más recientes estudios y revisiones sobre creatividad, como los de Said-Metwaly et al. (2017), Williams et al. (2016), Long et al. (2014), o el más lejano de Feist y Runco (1993), no se observa una atención específica a la relación de ésta con el ámbito deportivo. Y sucede así, aunque sean de un considerable tamaño, pues han sido tomados en cuenta cerca de 200 keywords en uno de ellos, o casi 2.000 artículos en otro. Es decir, a pesar que, desde el mundo del deporte se ha prestado una cierta atención a la creatividad (Martínez-Vidal y Díaz-Pereira, 2008, Tedesco da Costa Trevisan et al. 2018), no parece haber sido igualmente así en sentido contrario. Y salvo Mark Runco (Runco, y Pritzker, 2011; Richard et al., 2017; Richard, y Runco, 2020), pocos de los autores que son referentes en la psicología de la creatividad, han dedicado su interés hacia las implicaciones de ésta en el universo del deporte.

En la presente revisión sistemática, se busca aportar el recorrido que ha tenido el constructo psicológico de la creatividad en los últimos 25 años dentro del contexto 


\section{García-Peñas et al.}

deportivo. Se ha tomado éste en toda su extensión (deporte, competición, educación, entrenamiento, etc.), con el fin de realizar un acercamiento de la manera más amplia posible. Del mismo modo, se ha considerado la creatividad en todas sus modalidades (pensamiento divergente, creatividad motriz, producto creativo, etc.). Este documento puede servir de apoyo a futuros investigadores para conocer no solo que áreas de ambas temáticas han sido ya exploradas por los distintos autores en sus investigaciones, sino que también servirá como una guía de los principales hallazgos obtenidos en los estudios descritos.

Por tanto, el presente trabajo tiene como objetivo realizar una investigación de carácter exploratorio sobre la bibliografía acumulada acerca de creatividad y deporte, con la finalidad de tratar de aportar una visión general, en un campo que ha sido básicamente explorado de manera fragmentada. Se trata de encontrar elementos compartidos por esos diferentes estudios y asimismo considerar la manera en que podrían quedar organizados los diferentes enfoques y agrupamientos que se evidencien.

Para ello, se utiliza la técnica de la revisión sistemática, de acuerdo a los parámetros metodológicos que se indican a continuación.

\section{MATERIAL Y MÉTODOS}

\section{Diseño de investigación}

Esta investigación se lleva a cabo mediante la metodología propia de una revisión sistemática de la literatura científica, similar a la metodología guiada por el modelo PRISMA (Preferred Reporting Items for Systematic reviews and Meta-Analyses) (Urrútia y Bonfill, 2010). Para ello, se han tenido en cuenta todos los artículos que tratarán la temática de 'creatividad y deporte' de manera conjunta, en un periodo comprendido desde 1994 hasta 2019.

Dado que la temática de estudio es relativamente novedosa, y ha sido poco cubierta por los investigadores dentro de la literatura científica, se ha decidido mantener al margen algunos criterios de calidad más exhaustivos, que hubieran llevado a excluir algunos de los documentos hallados. Esta decisión fue tomada puesto que uno de los principales objetivos del trabajo era el de abarcar todos los documentos centrados en esta temática, independientemente de su nivel de calidad metodológica, y de esta forma exponer los principales hallazgos de dichos estudios, y, al mismo tiempo, destacar de manera general sus posibles flaquezas dentro de la discusión, proponiendo aspectos a mejorar en futuras investigaciones.

\section{Criterios de inclusión}

Para la selección de documentos se han tenido en cuenta los siguientes criterios de inclusión:

- Los documentos seleccionados deberán haber sido publicados en una franja temporal desde 1994-2019, cubriendo un espacio temporal de 25 años.

- Los estudios deberán estar en Español, Inglés o Portugués, descartando documentos en cualquier otro idioma.

- Deberá haber sido usado mínimo, un instrumento o técnica de evaluación propia del constructo de la creatividad.

- Ser una publicación científica en formato artículo revisado por pares.

- Los estudios deberán tener un carácter empírico, donde exista una muestra de individuos deportistas bien definida como tal por los investigadores.

\section{Bases de datos}

Los estudios deberán aparecer reflejados en las búsquedas derivadas de las bases de datos y revistas científicas que se va a exponer en la tabla 1.

Adicionalmente, se ha incluido una revista específica para la búsqueda, dado su carácter altamente especializado, su cobertura internacional y el hecho de que, a pesar de su gran relevancia en el área, no se encuentra suficientemente indizada en las referidas bases de datos.

\section{Palabras clave}

Para una búsqueda más exhaustiva se usaron las siguientes combinaciones de raíces léxicas que permitieron abarcar una mayor cantidad de términos referidos a creatividad y deporte: 'Creativi* AND Deport*','Creativ* AND Atlet*', 'Creativ* AND Sport*', '*Creativ* AND Athl*', 'Criativ* AND Espor*' y 'Criativ* AND Atlet*. 


\section{Creatividad y deporte}

Tabla 1

Principales bases de datos, subbases de datos y revistas revisadas.

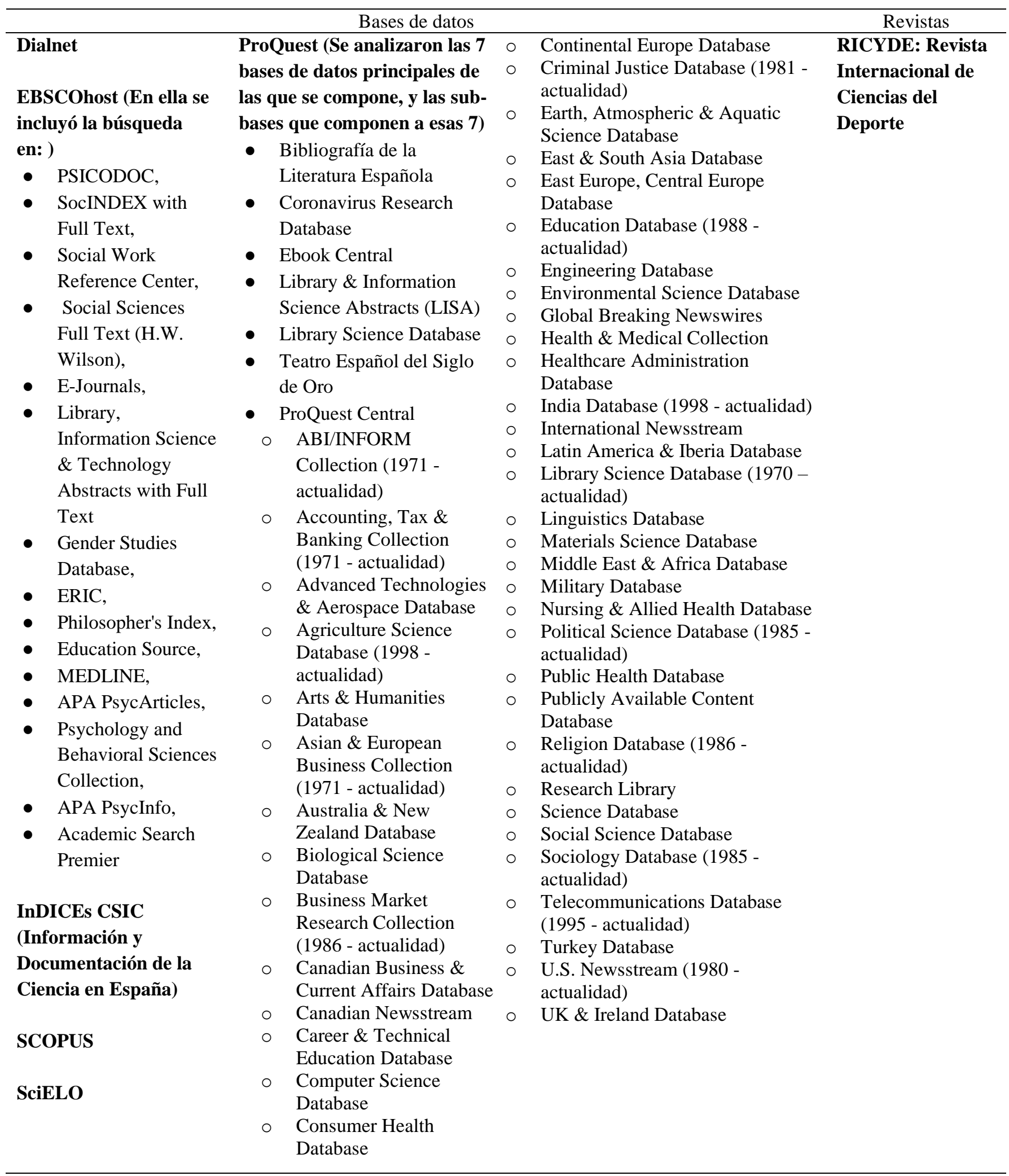




\section{García-Peñas et al.}

\section{Procedimiento}

Se realizó la búsqueda de artículos en las bases de datos mencionadas en la tabla anterior, y sólo se seleccionaron aquellos que incluyeran las palabras clave dentro del título, resumen o palabras clave del propio documento.

Se extrajo de cada estudio el año de publicación, idioma de estudio, el instrumento de creatividad utilizado, así como el número de la muestra, el sexo de los participantes, la franja de edad comprendida de la propia muestra y el país de procedencia de la misma.

\section{Plan de análisis}

Para que esta revisión sistemática pudiera clasificarse como descriptiva cualitativa, se ha buscado dar respuesta a cuestiones como ¿Cuántos estudios hay relacionados con la temática creatividad-deporte? ¿Qué tipo de muestra es la más común? ¿Cuáles son los instrumentos de creatividad más utilizados en este ámbito? ¿Qué tipo de deportes son más prevalentes en este tipo de estudios? y, aún con más importancia y siendo la información que más interés pudiera despertar en la comunidad científica, se ha dado respuesta a ¿Cuáles son los principales hallazgos obtenidos por los autores de dichos estudios? Para su exposición se han redactado dichos hallazgos, así como el resto de los datos de los estudios, en las tablas ofrecidas en el apartado de resultados.

\section{Diagrama de flujo}

En el siguiente diagrama de flujo, presente en la figura 1 , se puede observar cómo se pasó de 5,174 resultados prometedores, a tan solo 39 documentos que realmente cumplían con los criterios propuestos para esta revisión. Para alcanzar esos resultados, se realizó la identificación y cribado del groso total de los estudios siguiendo unos pasos similares a los de cualquier revisión sistemática guiada por la metodología PRISMA (Urrútia y Bonfill, 2010).

\section{Figura 1.}

Diagrama de Flujo.

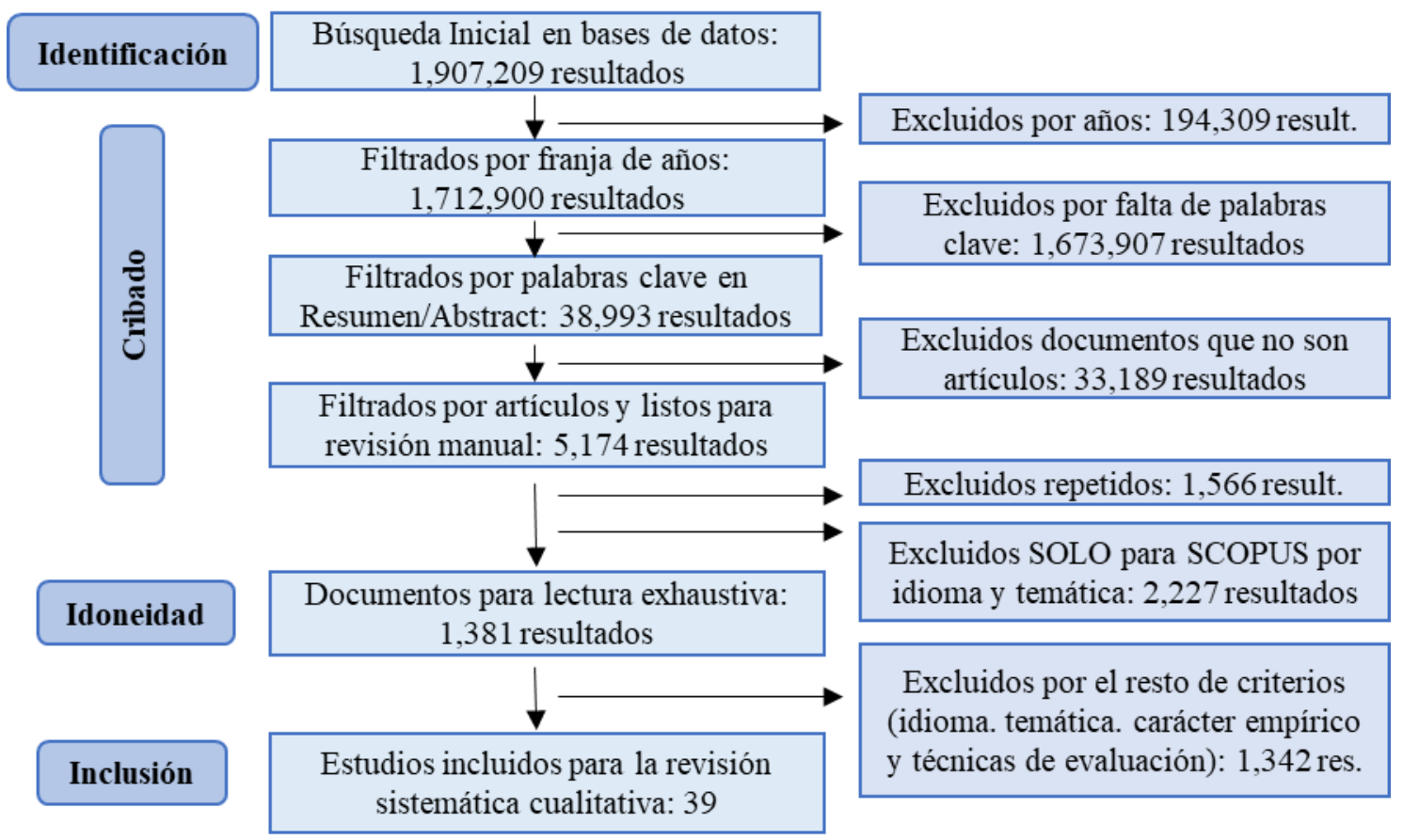




\section{Creatividad y deporte}

\section{RESULTADOS}

Una vez analizadas todas las bases de datos, y habiendo reducido el número de estudios a una cantidad de 5,174 resultados, se ha procedido a aplicar una revisión manual que permitiera, tras su lectura, aplicar los criterios de inclusión antes mencionados, dejando así, un total de 39 documentos que se van a nombrar y describir en las tablas 2, tabla 3 y tabla 4, englobando en cada tabla los documentos que se desprendían de cada una de las bases de datos que han sido extraídos.

\section{Tabla 2}

Documentos extraídos de Dialnet.

1 - Documento: Méndez-Martínez, E., y Fernández-Río, J. (2019). Diseño y validación de un instrumento de medición de la creatividad motriz en adolescentes. Revista Internacional De Medicina Y Ciencias De La Actividad Física Y Del Deporte, 19(75), 535.

- País de realización: España.

- Instrumentos de Creatividad: Jurado de 6 expertos en creatividad que evaluaron fluidez, flexibilidad, imaginación y originalidad a través de la expresión corporal en pruebas individuales.

- Muestra: 158 varones y 154 mujeres de entre 11 y 16 años.

- Estilo de deporte: (s/d*).

- Hallazgos alcanzados: Apoyo de la existencia del constructo de Creatividad Motriz (ICM) que puede resultar muy útil en futuras investigaciones entre psicología, deporte y creatividad.

2 - Documento: Chacón Araya, Yamileth, y Moncada Jiménez, José (2006). Relación entre personalidad y creatividad en estudiantes de educación física. Revista Electrónica "Actualidades Investigativas en Educación", 6(1).

- País de realización: Costa Rica.

- Instrumentos de Creatividad: CREA. Inteligencia Creativa.

- Muestra: 20 mujeres y 20 hombres matriculados en $1^{\circ}$ de Educación Física y Deportes con una edad de $(\overline{\mathrm{X}}=23.23 \pm$ 2.20 años) y 15 mujeres y 20 hombres en $4^{\circ}$ de la misma carrera con una edad de ( $\bar{X}=19.20 \pm 2.52$ años $)$.

- Estilo de deporte: (s/d*).

- Hallazgos alcanzados: Los alumnos de $4^{\circ}$ curso tenían una creatividad mayor, habiendo una relación significativa y directa entre la edad del estudiante y su creatividad. Debe tenerse en cuenta que esta diferencia en creatividad queda contrastada entre los alumnos de $1^{\circ}$ y $4^{\circ}$ curso, es por ello que se debe tener en consideración el papel de la educación que han recibido por los docentes y que les ha permitido ir estimulando su pensamiento divergente a lo largo de la carrera.

3 - Documento: Bohórquez-Gómez-Millán, M., y Checa-Esquiva, I. (2019). Desarrollo de competencias mediante ABP y evaluación con rúbricas en el trabajo en grupo en Educación Superior. REDU. Revista De Docencia Universitaria, 17(2),

- País de realización: España.

- Instrumentos de Creatividad: Escala de Potencial Creativo y de la Creatividad Practicada en el trabajo y en la organización (CPPC-17).

- Muestra: 37 alumnos del grado en Ciencias de la Actividad Física y el Deporte entre 18 y 25 años (n/a sexo**).

- Estilo de deporte: (s/d*).

- Hallazgos alcanzados: Los resultados mostraron que la experiencia del ABP no influyó en el potencial creativo de los alumnos, tampoco en el ejercicio de aplicación creativa, ni en el apoyo para la creatividad percibida.

${ }^{*} \mathrm{~s} / \mathrm{d}$ Sin datos. ${ }^{* *}$ n/a No hay datos de... 


\section{García-Peñas et al.}

Tabla 3

Documentos extraídos de EBSCOhost.

4 - Documento: Hüttermann, S., Memmert, D., y Nerb, J. (2019). Individual differences in attentional capability are linked to creative decision making. Journal Of Applied Social Psychology, 49(3), 159-167.

- País de realización: Alemania.

- Instrumentos de Creatividad: The football-specific divergent thinking task de Memmert

- Muestra: 24 jugadores de fútbol hombres entre 19 y 32 años.

- Estilo de deporte: Deporte de equipo.

- Hallazgos alcanzados: La experiencia y la capacidad de atención horizontal son buenos predictores del rendimiento en la toma de decisiones creativas de los jugadores de fútbol. Sin embargo, especialmente la relación entre la capacidad de atención de los jugadores de fútbol y sus decisiones creativas aún no permite ninguna conclusión causal sobre los mecanismos subyacentes que afectan la toma de decisiones de los participantes.

5 - Documento: Pepe, O. (2018). The Impact of The Creativity Levels of The Students Who Study at The School of Physical Education and Sport on Their Critical Thinking Dispositions. World Journal Of Education, 8(5), 185.

- País de realización: Turquía.

- Instrumentos de Creatividad: The Creativity Level Scale - The "How Creative Are You?" escala creativa desarrollada por Raudsepp

- Muestra: 189 hombres y 152 mujeres de entre 18 a 29 años pertenecientes a la universidad de educación física y deporte de Erciyes.

- Estilo de deporte: (s/d*).

- Hallazgos alcanzados: Se encontró que las puntuaciones de escala de las características de pensamiento crítico y creatividad estaban en un nivel promedio entre los participantes.

6 - Documento: Top, E., y Akil, M. (2018). Effects of the Sports on the Personality Traits and the Domains of Creativity. World Journal Of Education, 8(3), 56.

- País de realización: Turquía.

- Instrumentos de Creatividad: "Kaufman Domains of Creativity Scale" (K-DOCS).

- Muestra: 185 mujeres con una edad de $(\overline{\mathrm{X}}=21.42 \pm 2.20$ años $)$ y 408 hombres con una edad de $(\overline{\mathrm{X}}=22.05 \pm 2.22$ años $)$ pertenecientes a la Universidad de Uşak.

- Estilo de deporte: Deportes individuales y grupales.

- Hallazgos alcanzados: las estudiantes mujeres son más neuróticas. Las personas que practican deportes son más extrovertidas en comparación con las que no practican deportes, y los alumnos varones tienen puntuaciones más altas en los dominios de creatividad académica, creatividad mecánica científica, creatividad artística y psicoticismo en comparación con las puntuaciones de las alumnas mujeres.

7 - Documento: Aydin, E. (2019b). The Effect of Spiritual Intelligence and Optimistic on Creativity in Team and Individual Sports Activities of Trabzon University Students. Asian Journal Of Education And Training, 5(3), 397402.

- País de realización: Turquía.

- Instrumentos de Creatividad: Test de Torrance.

- Muestra: 148 alumnos de la universidad de Trabzon (n/a sexo y edad **)

- Estilo de deporte: 74 atletas en deportes de equipo y 74 atletas en deportes individuales.

- Hallazgos alcanzados: Hubo una diferencia significativa entre la mayor creatividad en las actividades deportivas de los estudiantes en el grupo de deportes de equipo frente a una creatividad menor mostrada por los deportistas de deportes individuales.

${ }^{*} \mathrm{~s} / \mathrm{d}$ Sin datos. ${ }^{* *} \mathrm{n} / \mathrm{a}$ No hay datos de... 


\section{Creatividad y deporte}

Tabla 3

(Continuación) Documentos extraídos de EBSCOhost.

8 - Documento: Aydin, E. (2019a). The Effect of Cultural Intelligence and Creative Thinking on the Practical Technical Capabilities of Trabzon University Physical Education Students. Asian Journal Of Education And Training, 5(2), 392-396.

- País de realización: Turquía

- Instrumentos de Creatividad: Test de Torrance.

- Muestra: 120 estudiantes de la universidad de Trabzon (n/a sexo y edad**)

- Estilo de deporte: 80 deportistas de equipo y 40 deportistas individuales.

- Hallazgos alcanzados: La inteligencia Cultural y el Pensamiento Creativo tuvieron un efecto positivo significativo en las capacidades técnicas y prácticas de los estudiantes de educación física de la Universidad de Trabzon que participaron en las lecciones prácticas (fútbol, voleibol y bádminton). Las tres lecciones prácticas incluidas la Inteligencia Cultural y el Pensamiento Creativo tuvieron un efecto positivo significativo en las habilidades técnicas de los estudiantes.

9 - Documento: Klatt, S., Noël, B., Musculus, L., Werner, K., Laborde, S.,y Lopes, M. et al. (2019). Creative and Intuitive Decision-Making Processes: A Comparison of Brazilian and German Soccer Coaches and Players. Research Quarterly For Exercise And Sport, 90(4), 651-665.

- País de realización: Alemania y Brasil.

- Instrumentos de Creatividad: Soccer-specific option-generation task.

- Muestra: 56 hombres entre 15 y 19 años.

- Estilo de deporte: Deporte de equipo.

- Hallazgos alcanzados: La relación entre creatividad e intuición muestra que el nivel de creatividad de los jugadores se asoció con la calidad de la primera opción generada. Los resultados enfatizan que los jugadores creativos también pueden considerarse buenos tomadores de decisiones intuitivas.

10 - Documento: Santos, S., Jiménez, S., Sampaio, J., y Leite, N. (2017). Effects of the Skills4Genius sports-based training program in creative behavior. PLOS ONE, 12(2), e0172520.

- País de realización: España.

- Instrumentos de Creatividad: Test de Torrance.

- Muestra: 40 niños de primaria (n/a sexo**) entre 8 y 10 años. A 22 de ellos se les aplica un programa con actividades físicas 5 meses.

- Estilo de deporte: $\left(\mathrm{s} / \mathrm{d}^{*}\right)$.

- Hallazgos alcanzados: Este estudio demostró que un programa de entrenamiento basado en el pensamiento creativo, la práctica diversificada, la alfabetización física y la pedagogía no lineal tiene un gran potencial para fomentar la creatividad general en los deportes de equipo.

11 - Documento: Noorafshan, L., Fazli, F., y Tavousisimakani, R. (2016). MSCwellbeing of the athletes. International Journal Of Pharmaceutical Research y Allied Sciences, 5(3), 448-457.

- País de realización: Irán.

- Instrumentos de Creatividad: 'Creativity Questionnaire', desarrollado por Abedi en Persia basado en la creatividad del Test de Torrance.

- Muestra: 150 hombres y 150 mujeres de clubs deportivos (n/a edad**).

- Estilo de deporte: (s/d*).

- Hallazgos alcanzados: Varios aspectos de la creatividad, a saber, la originalidad, la elaboración y la fluidez, están relacionados de manera significativa con el bienestar subjetivo percibido por los deportistas.

\footnotetext{
$*_{\mathrm{s}} / \mathrm{d}$ Sin datos. $* * \mathrm{n} / \mathrm{a}$ No hay datos de...
} 


\section{García-Peñas et al.}

Tabla 3

(Continuación) Documentos extraídos de EBSCOhost.

12 - Documento: Zoljanahi, E., Mohammadi-nezhade, M., Sadeghpour Gilde, B.,y Taheri, H. (2018). Investigating Sport Attributional Style, Sport Self-Efficacy, and Creativity in Elite Team Athletes. Iranian Journal Of Psychiatry And Behavioral Sciences, In Press(In Press).

- País de realización: Irán.

- Instrumentos de Creatividad: ‘Creativity Questionnaire', desarrollado por Abedi en Persia basado en la creatividad del Test de Torrance.

- Muestra: 863 mujeres 1.990 hombres. después se seleccionaron al azar 121 Hombres y 71 mujeres de 18 a 30 años.

- Estilo de deporte: Deporte de equipo.

- Hallazgos alcanzados: La autoeficacia deportiva tuvo un papel mediador positivo entre los estilos atribucionales deportivos y la creatividad.

13 - Documento: Orth, D., McDonic, L., Ashbrook, C., y van der Kamp, J. (2019). Efficient search under constraints and not working memory resources supports creative action emergence in a convergent motor task. Human Movement Science, 67, 102505.

- País de realización: Países Bajos.

- Instrumentos de Creatividad: Indicadores de creatividad.

- Muestra: 16 mujeres y 26 hombres de edad ( $\bar{x}=26.8 \pm 7.4$ años)

- Estilo de deporte: Deportes individuales.

- Hallazgos alcanzados: Las acciones creativas pueden estar relacionado con el enfoque inicial de la tarea, así como con la estrategia motora de cambiar activamente acciones durante la búsqueda de soluciones. Los aprendices que cambiaban cualitativamente de forma activa a diferentes patrones de coordinación de las extremidades o el uso de cada extremidad tendían a encontrar resultados más funcionales (efectivos). Este último punto se ve favorecido porque las acciones creativas fueron de alguna manera un producto de la tendencia a variar las acciones con mayor frecuencia en relación con el número total de acciones.

14 - Documento: Bowers, M., Green, B., Hemme, F., y Chalip, L. (2014). Assessing the Relationship Between Youth Sport Participation Settings and Creativity in Adulthood. Creativity Research Journal, 26(3), 314-327.

- País de realización: Estados Unidos

- Instrumentos de Creatividad: Torrance, ATTA por sus siglas en inglés (Abbreviated. Torrance Test for Adults).

- Muestra: 64 hombres y 36 mujeres entre los 19 y los 33 años. Pertenecientes a divisiones deportivas.

- Estilo de deporte: Deportes individuales y grupales.

- Hallazgos alcanzados: Los resultados proporcionan una clara evidencia de la relación entre la práctica de deportes informales en entornos no estructurados y el desarrollo de la creatividad. Según los participantes de este estudio, existe una relación positiva directa entre el tiempo dedicado a practicar deportes informales en la niñez y sus niveles de creatividad, fluidez, originalidad, elaboración y flexibilidad en general como adultos. La relación más fuerte entre el tiempo dedicado a la práctica de deportes informales y la creatividad se observó en el desarrollo de la flexibilidad creativa.

15 - Documento: Hendry, D., Williams, A., y Hodges, N. (2018). Coach ratings of skills and their relations to practice, play and successful transitions from youth-elite to adult-professional status in soccer. Journal Of Sports Sciences, 36(17), 2009-2017.

- País de realización: Reino Unido.

- Instrumentos de Creatividad: Las habilidades creativas fueron definidas por el estilo general y originalidad al tomar decisiones y mostrar habilidades inusuales (Memmert et al., 2010 citado en Hendry et al., 2018).

- Muestra: 102 hombres jugadores de elite de futbol entre los 13 y 15 años.

- Estilo de deporte: Deporte de equipo.

- Hallazgos alcanzados: Las horas de juego de fútbol no mostraron correlaciones esperadas con las calificaciones de habilidad táctica y creativa (o cualquier otra habilidad) en ningún momento. 


\section{Creatividad y deporte}

Tabla 3

(Continuación) Documentos extraídos de EBSCOhost.

16 - Documento: Furley, P., y Memmert, D. (2018). Can creative role models prime creativity in soccer players? Psychology Of Sport And Exercise, 37, 1-9.

- País de realización: Alemania

- Instrumentos de Creatividad: 'The football-specific divergent thinking task' de Memmert.

- Muestra: 60 hombres de edad ( $\overline{\mathrm{x}}=24.8, \mathrm{SD}=2.7$ años), y otros 60 hombres distintos de ( $\overline{\mathrm{X}}=23.0, \mathrm{SD}=2.6)$.

- Estilo de deporte: Deporte de equipo.

- Hallazgos alcanzados: El pensamiento creativo específico de un dominio se puede entrenar en jugadores de fútbol. La descripción inicial de un jugador de fútbol altamente creativo (Lionel Messi) en comparación con la descripción de un jugador de fútbol no creativo (Per Mertesacker o John Terry) resultó en una creatividad específica del fútbol. Las pruebas $t$ individuales sobre los componentes del índice de creatividad solo revelaron diferencias significativas para la fluidez y la flexibilidad, mientras que la originalidad no fue significativa.

- Documento: Cavallera, G., Boari, G., Labbrozzi, D., y Bello, E. (2011). Morningness-Eveningness Personality and Creative Thinking Among Young People Who Play Recreational Sport. Social Behavior And Personality: An International Journal, 39(4), 503-518.

- País de realización: Italia.

- Instrumentos de Creatividad: Test de Torrance.

- Muestra: 33 hombres y 26 mujeres entre los 19 y 31 años.

- Estilo de deporte: (s/d*).

- Hallazgos alcanzados: Las personas que dedican más tiempo al deporte recreativo tienen puntuaciones altas de pensamiento creativo. Con referencia a la elaboración, y las tipologías de personalidad parecen estar involucradas de manera diferente ya sea en el tiempo dedicado a las actividades deportivas o en las áreas de creatividad. Esto podría confirmar que el pensamiento creativo es un modelo complejo, en el que las variables se entremezclan con aspectos externos e internos, así como situaciones.

18 - Documento: Memmert, D., Baker, J., y Bertsch, C. (2010). Play and practice in the development of sport-specific creativity in team ball sports. High Ability Studies, 21(1), 3-18.

- País de realización: Alemania

- Instrumentos de Creatividad: Criterios de Creatividad Táctica

- Muestra: 30 mujeres y 42 hombres atletas con una edad de ( $\overline{\mathrm{X}}=23.2 \pm 4.4$ años).

- Estilo de deporte: Deporte de equipo.

- Hallazgos alcanzados: Los resultados indican que los jugadores más creativos acumularon más tiempo entrenando para su deporte principal que sus contrapartes menos creativas. Además, se informaron diferencias significativas entre estos grupos en cuanto al tiempo dedicado a actividades de juego no estructuradas. Ambos resultados sugieren que las experiencias de práctica y el juego temprano son influencias importantes en el desarrollo de la creatividad deportiva.

19 - Documento: Meric, E. (2014). The analysis of the thinking styles and creativity of the sports students studying in the different fields of university. Educational Research And Reviews, 9(20), 866-871.

- País de realización: Turquía

- Instrumentos de Creatividad: 'Thinking Styles Inventory' / Una adaptación de 'The Creativity Scale kirton inventory'.

- Muestra: 75 hombres y 61 mujeres con una edad media de 21,45 años.

- Estilo de deporte: Deporte de equipo.

- Hallazgos alcanzados: Se presentan relaciones significativas entre la creatividad y los estilos de pensamiento. Los hombres son más creativos que las mujeres. Se ha demostrado en este estudio que los estudiantes varones han usado casi todos los estilos de pensamiento más que las mujeres. Las participantes femeninas sólo han usado el estilo de pensamiento judicial más que los hombres.

\footnotetext{
*s/d Sin datos.
} 


\section{García-Peñas et al.}

Tabla 3

(Continuación) Documentos extraídos de EBSCOhost.

20 - Documento: Tohănean, I. (2016). Study on the level of creativity for the female handball players (Seniors). Series IX: Sciences Of Human Kinetics, 9(2), 69-74.

- País de realización: Rumania

- Instrumentos de Creatividad: 'Questionnaire of Creative Attitudes' / 'The Test of Imagination and Creativity' / 'Test of Identification the Creativity Coefficient'.

- Muestra: 40 mujeres deportistas entre los 18 y 27 años.

- Estilo de deporte: Deporte de equipo.

- Hallazgos alcanzados: Los resultados mostrados por las jugadoras de balonmano han mostrado un potencial creativo altamente significativo.

21 - Documento: Memmert, D. (2006). Developing creative thinking in a gifted sport enrichment program and the crucial role of attention processes. High Ability Studies, 17(1), 101-115.

- País de realización: Alemania.

- Instrumentos de Creatividad: Criterio de expertos en creatividad.

- Muestra: 25 hombres y 8 mujeres con una edad de ( $\overline{\mathrm{X}}=8.2, \mathrm{SD}=1.09$ años $)$

- Estilo de deporte: Deporte de equipo.

- Hallazgos alcanzados: El programa de enriquecimiento deportivo mostró un alto aumento del pensamiento creativo entre los niños superdotados.

22 - Documento: Vestberg, T., Gustafson, R., Maurex, L., Ingvar, M., y Petrovic, P. (2012). Executive Functions Predict the Success of Top-Soccer Players. Plos ONE, 7(4), e34731.

- País de realización: Suecia.

- Instrumentos de Creatividad: 'D-KEFS test battery of executive functions '/ 'Desing Fluency (DF)'.

- Muestra: 31 hombres y 26 mujeres con una edad de ( $\overline{\mathrm{X}}=25.3 ; \mathrm{SD}=4.2$ divisiones altas) y $(\overline{\mathrm{X}}=22.8 ; \mathrm{SD}=4.1$ años en divisiones bajas). Todos eran jugadores de futbol profesional en sus distintas divisiones.

- Estilo de deporte: Deporte de equipo.

- Hallazgos alcanzados: Las funciones ejecutivas generales son importantes en el fútbol e incluso puede predecir un futuro éxito en los jugadores de fútbol. Los jugadores de fútbol en el grupo de la EH tenían importantes mejores resultados que los jugadores de fútbol en el grupo de la LD.

23 - Documento: Veraksa, A., y Gorovaya, A. (2012). Imagery training efficacy among novice soccer players. Procedia - Social And Behavioral Sciences, 33, 338-342.

- País de realización: Rusia

- Instrumentos de Creatividad: Versión Rusa del ‘The Sport Imagery Questionnaire’ (SIQ) / Test de Torrance

- Muestra: 41 hombres jugadores de fútbol con una edad de ( $\bar{X}=10.8$ años)

- Estilo de deporte: Deporte de equipo.

- Hallazgos alcanzados: Se concluye que un aumento en la cantidad de diferentes imágenes mentales que realizan los jugadores sobre una situación de juego está estrechamente vinculado con un aumento considerables de los resultados en el rendimiento deportivo. 


\section{Creatividad y deporte}

Tabla 3

(Continuación) Documentos extraídos de EBSCOhost.

24 - Documento: Bernacka, R., Sawicki, B., Mazurek-Kusiak, A., y Hawlena, J. (2016). Conforming and nonconforming personality and stress coping styles in combat athletes. Journal Of Human Kinetics, 51(1), 225-233.

- País de realización: Polonia.

- Instrumentos de Creatividad: 'The KANH III (Creative Behaviour Questionnaire III)'

- Muestra: 346 hombres que practicaban deportes ( $\overline{\mathrm{X}}=22 \pm 3.5$ años $)$

- Estilo de deporte: Deporte individual

- Hallazgos alcanzados: Los análisis de las diferencias de estrés los estilos de afrontamiento (conforme/no conforme) mostraron que los inconformes eran más propensos a utilizar el sistema de tareas estilo de afrontamiento. La inconformidad como un rasgo de personalidad puede, en realidad, ser un buen pronosticador para hacer frente a las tareas el estrés que muestran los atletas de combate. La comprensión de la función innovadora de el inconformismo y el estilo de afrontamiento orientado a las tareas reflejado en el rendimiento del competidor podría ser más claro si analizamos la función del mecanismo de la personalidad (Bernacka, 2009 citado en Bernacka. et al., 2016) en la a la luz de la teoría de la actitud creativa (Grohman y Schmidt, 2013 citado en Bernacka. et al., 2016). La personalidad no conforme es abierto y flexible en la etapa de "creativo exploración". Los rasgos mencionados anteriormente sobre el mecanismo del (no) conformismo (Bernacka, 2009 citado en Bernacka. et al., 2016) debería ser interpretados como signos del uso individual de su personalidad creativa, que se hace difícil por algunos factores subjetivos externos. Debería ser inspirador para los entrenadores y el deporte psicólogos para identificar a los competidores con talento teniendo en cuenta sus habilidades psicológicas que juegan un papel importante en el rendimiento deportivo y para emplear adecuadamente su potencial creativo para la transgresión, lo que también es factible en el combate deportes.

25 - Documento: Memmert, D. (2011a). Creativity, expertise, and attention: Exploring their development and their relationships. Journal Of Sports Sciences, 29(1), 93-102.

- País de realización: Alemania.

- Instrumentos de Creatividad: Tarea específica de pensamiento divergente / Tarea no específica de pensamiento divergente, de la subprueba perteneciente a 'Divergent Thinking Test'.

- Muestra: 90 hombres y 30 mujeres con edades entre los 7 y 13 años.

- Estilo de deporte: Deporte de equipo.

- Hallazgos alcanzados: Todos los hallazgos respaldan el hecho de que los procesos de atención y los efectos de la experiencia desempeñan un papel en el desarrollo del pensamiento creativo no específico y específico.

- Documento: Memmert, D., y Perl, J. (2009). Game creativity analysis using neural networks. Journal Of Sports Sciences, 27(2), 139-149.

- País de realización: Alemania.

- Instrumentos de Creatividad: Criterio de expertos evaluando Originalidad y Flexibilidad.

- Muestra: 65 hombres y 25 mujeres con una edad de ( $\overline{\mathrm{X}}=6.8, \mathrm{SD}=0.8$ años).

- Estilo de deporte: Deporte de equipo.

- Hallazgos alcanzados: Los niños del grupo entrenado en hockey sobre césped mostraron una fluctuación de arriba hacia abajo más fuerte que el grupo de fútbol y viceversa. No se observaron diferencias marcadas en los otros tres comportamientos de aprendizaje (estable, aumento o disminución en la creatividad del juego) entre los tres grupos.

27 - Documento: Memmert, D., y Roth, K. (2007). The effects of non-specific and specific concepts on tactical creativity in team ball sports. Journal Of Sports Sciences, 25(12), 1423-1432.

- País de realización: Alemania.

- Instrumentos de Creatividad: ‘Game Test Situation (GTS)' Desarrollado por Memmert para evaluar la creatividad.

- Muestra: 159 hombres y 80 mujeres con una edad de ( $\overline{\mathrm{x}}=6.9, \mathrm{SD}=0.83$ años).

- Estilo de deporte: Deporte de equipo.

- Hallazgos alcanzados: Todos los tratamientos resultaron en un aumento en los valores característicos de la creatividad. Los datos generados en este estudio apoyan una transferencia entre diferentes creatividades específicas o la idea de una la creatividad general independiente de los juegos de pelota específicos permanece abierto. En conclusión, los datos tienden a apoyar la opinión de que los conceptos no específicos y específicos son similares en términos de desarrollo de la creatividad. 


\section{García-Peñas et al.}

Tabla 3

(Continuación) Documentos extraídos de EBSCOhost.

28 - Documento: Memmert, D. (2010). Testing of tactical performance in youth elite soccer. Journal Of Sports Science And Medicine, 9, 1-7.

- País de realización: Alemania.

- Instrumentos de Creatividad: 'Game Test Situation (GTS)' Desarrollado por Memmert para evaluar la creatividad. / 12 Medidas de rendimiento para la creatividad evaluadas por un criterio de expertos.

- Muestra: 195 individuos (n/a sexo**) con una edad entre 18 y 21 años.

- Estilo de deporte: Deporte de equipo.

- Hallazgos alcanzados: Los instrumentos pueden ser aplicados para capturar la creatividad específica del fútbol y la inteligencia de juego (diferencia lo convergente de lo divergente). Los resultados con respecto al pensamiento táctico divergente mostraron claramente que se observaron procesos de cambio muy diferentes en los jugadores de la DFB.

29 - Documento: Thomson, P., y Victoria-Jaque, S. (2016). Visiting the Muses: Creativity, Coping, and PTSD in Talented Dancer and Athletes. American Journal Of Play, 8(3), 363-378.

- País de realización: Estados Unidos.

- Instrumentos de Creatividad: 'The experience of creativity questionnaire (ECQ)'

- Muestra: 72 hombres y 136 mujeres con una edad de ( $\overline{\mathrm{X}}=23.59, \mathrm{SD}=5,82$ años $)$

- Estilo de deporte: $\left(\mathrm{s} / \mathrm{d}^{*}\right)$

- Hallazgos alcanzados: Los hallazgos indican que participar en el proceso creativo, aunque provocó más ansiedad en el grupo de TEPT, permitió una profunda y significativa conexión durante la experiencia. Estas experiencias pueden promover la resiliencia manifestada en estos talentosos bailarines y atletas. Muchos estudios apoyan la implementación de la terapia de juego y la terapia de arte expresivo al tratar a niños traumatizados.

- Documento: Richard, V., Abdulla, A., y Runco, M. (2017). Influence of Skill Level, Experience, Hours of Training, and Other Sport Participation on the Creativity of Elite Athletes. Journal Of Genius And Eminence, 2(1), 65-76.

- País de realización: Canadá.

- Instrumentos de Creatividad: 'Divergent thinking (DT)'. / 'Creative Attitude and Values'. / 'Creative personality How Would You Describe Yourself (HWYDY)' / 'Creative Activity and Accomplishment Checklist (CAAC)’.

- Muestra: 94 hombres y 114 mujeres de entre 14 y 37 años.

- Estilo de deporte: Deportes individuales y grupales.

- Hallazgos alcanzados: Los resultados de la presente investigación revelaron que los atletas expertos exhiben un mayor nivel de creatividad que los atletas intermedios y avanzados. Más específicamente, atletas expertos (p. ej., atletas olímpicos) mostraron un nivel significativamente más alto de flexibilidad cognitiva que sus contrapartes menos hábiles.

31 - Documento: Predoiu, R., Mitrache, G., y Predoiu, A. (2016). The effects of induced emotions on working memory, creativity, repetition speed and precision: a pilot study. Physical Education, Sport And Kinetotherapy Journal, 12(2), 44-51.

- País de realización: Rumania.

- Instrumentos de Creatividad: 'Creativity test' adaptado despues por Torrance y su equipo.

- Muestra: 38 hombres de entre 20 y 22 años.

- Estilo de deporte: Deportes individuales y grupales.

- Hallazgos alcanzados: El grupo sometido a emociones negativas tristes obtuvo una peor puntuación en la fluidez relacionada con la creatividad. Con respecto al grupo que fue sometido a emociones de miedo no hubo diferencias significativas en las puntuaciones de creatividad.

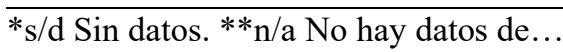




\section{Creatividad y deporte}

Tabla 3

(Continuación) Documentos extraídos de EBSCOhost

32 - Documento: S. Colzato, L., Szapora, A., Pannekoek, J., y Hommel, B. (2013). The impact of physical exercise on convergent and divergent thinking. Frontiers In Human Neuroscience, 7, 1-6.

- País de realización: Países Bajos.

- Instrumentos de Creatividad: Tarea de usos alternativos (Pensamiento Divergente).

- Muestra: 48 hombres y 48 mujeres con una edad ( $\bar{X}=20.6$ años).

- Estilo de deporte: (s/d*).

- Hallazgos alcanzados: Los resultados proporcionan un programa preliminar para vincular el ejercicio y la creatividad. Los no deportistas no se beneficiaron del ejercicio agudo. Para las personas que realizan ejercicios, la ausencia de ejercicio (descanso) perjudica la creatividad forma más que su presencia mejora. Nuestras observaciones sugieren que más ejercicio puede mejorar la convergencia de pensamiento, al menos en las personas con mayor grado de aptitud física.

- Documento: Furley, P., y Memmert, D. (2015). Creativity and working memory capacity in sports: working memory capacity is not a limiting factor in creative decision making amongst skilled performers. Frontiers In Psychology, 6 , $1-7$.

- País de realización: Alemania.

- Instrumentos de Creatividad: 'Creativity task', adaptaron el 'The soccer-specific divergent-thinking test'.

- Muestra: 61 hombres con una edad de ( $\overline{\mathrm{X}}=23.48, \mathrm{SD}=3.6$ años$)$.

- Estilo de deporte: Deporte de equipo.

- Hallazgos alcanzados: No encuentran ninguna relación directa entre la memoria de trabajo y la creatividad. Nuestros resultados sugieren que la función moderadora de la naturaleza de la actividad creativa juega un papel un papel importante en la interacción entre los pensamientos divergentes y la memoria de trabajo.

34 - Documento: Herman-Tofler, L., y Tuckman, B. (1998). The effects of aerobic training on children's creativity, selfperception, and aerobic power. SPORT PSYCHIATRY, 7(4), 773-790.

- País de realización: Estados Unidos.

- Instrumentos de Creatividad: Test de Torrance

- Muestra: 52 alumnos (n/a edad y sexo**)

- Estilo de deporte: $\left(\mathrm{s} / \mathrm{d}^{*}\right)$.

- Hallazgos alcanzados: Los resultados de este estudio demuestran algunos beneficios psicológicos del ejercicio físico para los niños de tercer grado. Las diferencias significativas encontradas en las puntuaciones posteriores a la prueba de la creatividad figurativa de los niños

*s/d Sin datos. **n/a No hay datos de...;

\section{Tabla 4}

Documentos extraídos de ProQuest.

35 Documento: Wen-Yu, C., y Jhao-You, H. (2017). A Study Of Creative Teaching And Technological Creativity Using Hierarchical Linear Models. The International Journal Of Organizational Innovation, 9(1), 60-73.

País de realización: Taiwán.

Instrumentos de Creatividad: 'Technological creativity: measured by the scale on technological creativity' desarrollada $\mathrm{Wu}$.

Muestra: 30 coaches y 285 atletas (n/a sexo y edad**)

Estilo de deporte: (s/d*)

Hallazgos alcanzados: Los resultados de este estudio indican que los atletas con mayor autoeficacia demuestran una mayor creatividad

${ }^{*} \mathrm{~s} / \mathrm{d}$ Sin datos. ${ }^{* *}$ n/a No hay datos de... 


\section{García-Peñas et al.}

\section{Tabla 4}

(Continuación) Documentos extraídos de ProQuest.

36 Documento: Rasmussen, L., Y Østergaard, L. (2016). The Creative Soccer Platform: New Strategies for Stimulating Creativity in Organized Youth Soccer Practice. Journal Of Physical Education, Recreation y Dance, 87(7), 9-19.

País de realización: Dinamarca.

Instrumentos de Creatividad: Criterio de expertos en la evaluación de la creatividad

Muestra: 15 hombres entre 13 a 14 años y 3 coaches de 27 años.

Estilo de deporte: Deporte de equipo.

Hallazgos alcanzados: Varias veces han tenido la energía extra necesaria para hacer lo inesperado durante los partidos. Han hecho algunas cosas después de las sesiones de entrenamiento de creatividad que no los había visto antes. Muestra más confianza. (Andy, entrenador del equipo juvenil) En resumen, TCSP puede demostrar que aumenta la autoeficacia creativa de los jugadores en el fútbol como se ha demostrado anteriormente que la Plataforma Creativa lo hace en entornos escolares.

37 Documento: Burdzicka-Wołowik, J., y Góral-Radziszewska, K. (2014). Selected Personality Traits of Women Training Combat Sports. Polish Journal Of Sport And Tourism, 21(1), 3-7.

País de realización: Polonia.

Instrumentos de Creatividad: 'The Creative Behaviour Questionnaire'.

Muestra: 199 mujeres de entre 17 y 36 años.

Estilo de deporte: Deporte individual.

Hallazgos alcanzados: La última prueba analizada fue el Cuestionario de Conducta Creativa. Debido al carácter de la publicación, se analizaron dos escalas de la prueba. Se revelaron diferencias estadísticamente significativas en lo que respecta a los comportamientos no conformistas. En este caso, los atletas que entrenaron deportes de combate tuvieron resultados significativamente más altos que las mujeres que no entrenaron ningún deporte. En la escala de comportamientos conformistas, los encuestados del segundo grupo lograron resultados más altos que los atletas; sin embargo, estas no fueron diferencias estadísticamente significativas.

38 Documento: Rutkowska, K., y Gierczuk, D. (2012). Selected Cognitive And Emotional Resources Of Untrained Youth And Young Wrestlers. Polish Journal Of Sport And Tourism, 19(3), 190-195.

País de realización: Polonia.

Instrumentos de Creatividad: The KANH III (Creative Behaviour Questionnaire III).

Muestra: 38 hombres y 40 mujeres en tono a los ( $\overline{\mathrm{X}}=17.2$ años).

Estilo de deporte: Deporte individual.

Hallazgos alcanzados: Sólo las luchadoras manifestaron bajos niveles de inteligencia emocional; sus niveles de inconformidad, pensamiento heurístico y actitud creativa también fueron más bajos que en las estudiantes.

39 Documento: Memmert, D. (2007). Can Creativity Be Improved by an Attention-Broadening Training Program? An Exploratory Study Focusing on Team Sports. Creativity Research Journal, 19(2-3), 281-291.

País de realización: Alemania.

Instrumentos de Creatividad: 'Game Test Situations (GTS)'.

Muestra: 56 hombres con una edad de ( $\overline{\mathrm{X}}=6.5, \mathrm{SD}=6$ años $)$.

Estilo de deporte: Deporte de equipo.

Hallazgos alcanzados: El grupo de entrenamiento para ampliar la atención mejoró considerablemente su desempeño creativo con el tiempo. El programa de ampliación de la atención puede jugar un papel útil para promover el desarrollo de la creatividad en los niños.

\section{Idioma de los estudios, revistas y años de publicación}

En referencia a la tabla principal de resultados, donde se pueden ver expuestos los 39 documentos que conforman esta revisión sistemática, de los cuales 36 de estos documentos, el $92.31 \%$, están publicados en inglés, mientras que tan solo 3 de ellos, el 8.33\%, están redactados en español.

En relación a las 30 revistas científicas que han publicado los 39 documentos expuestos en esta revisión sistemática, 'Journal Of Sports Sciences' es 


\section{Creatividad y deporte}

la revista que más documentos ha acogido de esta temática, encontrado en ella 4 artículos, el 10,26\% del total, seguida por 'Asian Journal Of Education And Training', 'Creativity Research Journal', 'High Ability Studies', 'PLOS ONE', 'Polish Journal Of Sport And Tourism' y 'World Journal Of Education', obteniendo de cada una de ella 2 artículos científicos, el $5.13 \%$ por cada una de ellas. Por último, se ha encontrado que del resto de las revistas, sólo se obtuvo 1 único documento por cada una de ellas, sobre la temática que se está abarcando.

Por ello, es necesario reflejar que el primer estudio que versa sobre la temática de 'deporte y creatividad', en esta revisión sistemática, data de 1998, siendo el siguiente más cercano de 2006. Desde entonces, ha habido aproximadamente 1 publicación por año hasta llegar a 2016, año en el cual se hicieron 6 publicaciones sobre esta temática, siendo sólo superado por el año 2019 en el cual se llegaron a hacer 7 publicaciones.

\section{Procedencia, deportes y tipos de muestras}

Con respecto a la procedencia de las muestras de cada estudio, se puede observar que 40 países conforman el total de naciones de procedencia de las distintas muestras, siendo Alemania, con un $27.5 \%$, el país al que más se recurrió para obtener muestras de atletas; seguido de Turquía con un $12.5 \%$. Mientras que Polonia, Estados Unidos y España, representan un 7.5\% cada uno; Rumania, Países Bajos e Irán un 5\% cada uno, y el resto un $2.5 \%$ respectivamente de los 9 países restantes.

En sólo 4 estudios de los 39, se utilizó una muestra compuesta únicamente por atletas de deportes individuales. Mientras que, en 18 estudios se utilizó atletas de deportes de equipo. En otros casos, en 6 estudios, los atletas practicaron deportes mixtos, tanto individuales como de equipo. No obstante, es necesario remarcar que, en 11 de los 39 estudios, en un $28.21 \%$ de los mismos, la muestra no quedaba definida en base al deporte que practicaban, sino que sólo se hacía mención a si practicaban regularmente, o habían practicado en los últimos meses, algún deporte.

La muestra total de participantes estaba compuesta en un $52.06 \%$ por hombres (2,790 participantes), mientras que el $31.01 \%$ eran mujeres $(1,662$ participantes). Nuevamente, se ha de hacer especial mención a que el 16.74\% (897 de los participantes), no quedaban definidos por sexo.

Por último, 10 de los estudios, un $25.64 \%$, estaban compuestos por menores de edad; 21 de los estudios, un $53.85 \%$ estaban compuestos por participantes mayores de edad. Mientras que, sólo 3 estudios, el $7.69 \%$ estaba compuesto tanto por participantes menores como por mayores de edad, y se debe remarcar que, en 5 estudios, el $12.82 \%$ de los documentos que componen esta revisión sistemática, no queda definida la edad de la muestra.

\section{Instrumentos de medición de la creatividad}

En lo referente a los tipos de mediciones que se realizaron para determinar el nivel de la creatividad de los participantes, encontramos que, en 7 de los 39 estudios, un $17.95 \%$ de los mismos, el tipo de medición se realizó a través de un criterio de expertos, los cuales evaluaban de manera visual cómo de creativos eran los sujetos, al analizar una jugada. En tan sólo 1 de los estudios se utilizó de manera conjunta el criterio de expertos y un instrumento para la medición de la creatividad, mientras que, en el resto de los estudios, 31 de ellos utilizaron tan sólo 1 instrumento para la medición de la creatividad, frente a 4 estudios que utilizaron 2 o más instrumentos para la medida de la misma.

De entre todos los instrumentos utilizados, el que mayor frecuencia de uso mostró entre los estudios seleccionados fue el 'Test de Torrance' siendo usado por los investigadores en 6 de los 32 estudios que utilizaron instrumentos, seguido por 'Game Test Situation (GTS)' el cual se utilizó en 3 de los 32 estudios. Mientras que con la misma frecuencia de aparición encontramos, en 2 de los 32 estudios se utilizó 'Abedi Creativity Questionnaire' , 'The KANH III (Creative Behaviour Questionnaire III)' y 'The football-specific divergent thinking'. Para finalizar, en el resto de los 21 estudios que se utilizaron instrumentos, éstos tenían una frecuencia de aparición de 1 , no repitiéndose el instrumento de medición en los demás estudios.

\section{Hallazgos alcanzados}

En cada uno de los 39 artículos finalmente seleccionados se ha incluido un apartado sobre hallazgos alcanzados en la investigación correspondiente (v. Tablas 2, 3 y 4). Se presenta ahora 


\section{García-Peñas et al.}

su consideración en conjunto y una clasificación de los mismos. Se organiza en cinco grupos de artículos, en función de su objeto y/o su contexto. Todos ellos, abordan cuestiones relativas a la Creatividad y el Deporte, consideradas en las siguientes categorías:

\section{1.- Estudios Descriptivos (6/39)}

Un pequeño grupo de artículos se centra en el concepto de creatividad motriz, y sus características, resaltando su utilidad en futuras investigaciones entre psicología, deporte y creatividad. Se trata de artículos de carácter genérico que pueden indagar en torno aspectos relativos sobre si la creatividad es más o menos genérica en el conjunto de los deportes, o si puede transferirse de unos a otros. Se podría decir que son artículos de creatividad que realizan una mirada básica a la implementación de este concepto en el deporte. Resultan preferentemente descriptivos, diferenciando entre grupos por sexos, por tipo de deporte, por su nivel como expertos, por aptitudes: forma física o por actitudes: conformismo vs. Inconformismo.

\section{2.- Estudios en Entornos Educativos (10/39)}

Un segundo grupo de artículos, el mayor de ellos, aborda cuestiones relativas al ámbito educativo, en el que la creatividad y el deporte se encuentran interrelacionadas. En general, tiende a considerarse la creatividad como una variable dependiente, que resulta estimulada y que tiende a mejorar cuando los alumnos practican deportes, sobre todo en niños de altas capacidades y en deportes de equipo. También hay algunos estudios en los que no se dan los citados efectos de mejora. Asimismo se comprueban efectos a la inversa, sobre la mejora en la técnica en estudiantes de educación física, tras seguir una formación en creatividad. Se exploran relaciones entre creatividad y un amplio conjunto de variables como edad, metodología ABP, pensamiento crítico, dominios educativos, personalidad, etc. En general aparecen efectos favorecedores en ambas direcciones, encontrando tanto rasgos más saludables y mejoras cognitivas entre los practicantes de deportes, como también la práctica deportiva cuando reciben formación en creatividad.

\section{3.- Estudios sobre Funciones Ejecutivas (7/39)}

Un tercer grupo de artículos bastante notable, va dirigido a estudiar la repercusión que tiene la creatividad en las funciones ejecutivas de los atletas.
Se trata en general de estudios basados en la capacidad de atención y en la toma de decisiones. Los atletas creativos son intuitivos y suelen tomar buenas decisiones en el terreno de juego. Asimismo, valoran el estilo de pensamiento que diferencia a hombres y mujeres atletas en la toma de decisiones. Las funciones ejecutivas están relacionadas con el éxito en jugadores de equipo pues son capaces de tener una red más amplia, captando mayor información del entorno y discerniendo sobre su relevancia. Por otro lado, un estudio sugiere que el tipo de actividad creativa es una variable moderadora entre pensamiento divergente y la memoria de trabajo. En esta línea, una prueba estudiada resulta capaz de diferenciar entre inteligencia y creatividad en el desarrollo del juego mismo, lo que le permite hacer una diferenciación convergente-divergente en la práctica del deporte.

\section{4.- Estudios sobre Entrenamiento (8/39)}

En este cuarto grupo de estudios, la creatividad tiende a ser entendida como un recurso en el juego que se puede potenciar. Por ello, se proponen programas de entrenamiento de la creatividad, evaluando pretestpostest, resultando significativas las diferencias encontradas. En un estudio se descompone la creatividad y se observa si incide en el rendimiento, encontrando que existen diferencias significativas en fluidez y flexibilidad; mientras que en originalidad no son significativas. Se aprecian relaciones entre entrenar en espacios externos o internos, o el tiempo dedicado a cada uno y el nivel de creatividad. Asimismo, se estudian efectos del entrenamiento sobre el número de imágenes mentales que los jugadores son capaces de plantearse en una situación de juego y su posterior rendimiento; también el aumento en nivel de autoeficacia creativa en jugadores de fútbol al visualizar combinaciones posibles de próximos movimientos en el juego; o se hace entrenamiento de potenciación de la atención y se da un mejor desarrollo de su creatividad. En general, el entrenamiento se muestra como favorecedor del desempeño creativo en el deporte, de modo particular si incluye programas específicos que promuevan su estimulación.

\section{5.- Estudios sobre Salud (8/39)}

En un quinto grupo de estudios, la creatividad es entendida como fortaleza en programas de promoción de la salud. Se considera que la originalidad, la elaboración y la fluidez, están relacionados de manera 


\section{Creatividad y deporte}

significativa con el bienestar subjetivo percibido por los deportistas. En general, se da una mejora de los estilos atribucionales para aumentar la autoestima, la autoeficacia, el bienestar psicológico, cambio de enfoque en los estilos de afrontamiento ante el estrés, reducción de la ansiedad, un mayor manejo emocional o aumentar la inteligencia emocional. También se aborda el uso de la terapia de juego y de arte expresivo como recursos ante el TEPT y la promoción de la resiliencia. La emoción de tristeza resulta afectar más que la de miedo a una pérdida de eficacia creativa. Y al contrario, se dan en varios estudios relaciones entre el ejercicio físico y ciertos beneficios psicológicos, entre ellos la creatividad. Se resalta un estudio que considera los beneficios que para el combate o la competición puede suponer la combinación de un potencial creativo y cierta tendencia al inconformismo asociada.

\section{DISCUSIÓN}

Los hallazgos en los 39 estudios seleccionados resultan muy dispersos, puesto que, salvo las propuestas de Memmert (2011b), no existe todavía una línea consolidada de definición de la creatividad en el contexto deportivo, ni de recursos para su evaluación. Se trata de hallazgos sobre interacciones relevantes, aunque limitadas a aspectos muy concretos. Se puede encontrar, por ejemplo, datos acerca de la eficacia en un programa de entrenamiento, o sobre los efectos positivos del deporte en la creatividad y en otras variables, en el contexto educativo. Pero fundamentalmente se trata de estudios descriptivos o que analizan interacciones restringidas a ciertas modalidades deportivas, a variables concretas o a diferencias por sexo, tipos de deporte etc. Asimismo, apenas hay alusiones a instrumentos o metodologías plenamente eficaces en la evaluación de la creatividad en la actividad deportiva. El concepto mismo de creatividad motriz, parece ser uno de los progresos más logrados en este campo de estudio, pero en general, cabe decir que se trata todavía de un área con un gran potencial de desarrollo.

Observando el recorrido de los estudios centrados en esta temática, se puede apreciar cómo, dentro de nuestra revisión sistemática, el primer estudio fue realizado por Herman-Tofler y Tuckman (1998), seguido de Chacón-Araya y Moncada-Jiménez (2006) y Memmert (2006) que 8 años después retomaron los estudios de creatividad y deporte. Desde entonces, el flujo de publicaciones sobre esta temática ha sido constante, aunque bajo, puesto que aproximadamente ha habido 1 publicación sobre esta materia al año, y la mayoría de estas investigaciones estaban dirigidas por Memmert (2006; 2007; 2010; 2011), Memmert y Perl (2009) hasta llegado 2012, año en el que otros nuevos autores se sumaron a labores de investigación centradas en esta área. No fue hasta 2016, donde por primera vez hubo 6 publicaciones sobre esta temática en un año, llegando 2019 a ser el año que más publicaciones se realizaron. Con ello, se puede observar que creatividad y deporte es un área de conjunto muy novedosa, siendo previsible que en los próximos años se vaya a realizar un mayor número de hallazgos en este campo (Putwain et al., 2012)

Diversos autores destacan la importancia de la creatividad a la hora de alcanzar la excelencia en los distintos campos formales del esfuerzo (Bowers et al., 2014; Memmert, 2015; Runco, 2004; Simonton, 2012). Incluso Bush y Salmela (2002) demostraron que la creatividad y la innovación eran esenciales para los atletas de alto rendimiento, y aún más dentro de sus años de mayor rendimiento deportivo. El propio Memmert (2015) afirmaba la importancia de la creatividad a la hora de que los atletas desarrollen nuevas estrategias y habilidades que les permita superponer sus resultados por encima de los del adversario, logrando encontrar soluciones creativas que el oponente no pueda anticipar. Algunos autores desde mediados del siglo XX introdujeron el concepto de creatividad motriz (Domínguez-Iglesias et al., 2014), haciendo referencia a la resolución de problemas y a la expresión de una idea o sentimiento a través de los movimientos del cuerpo (Karaca y Aral, 2017). Dicho concepto, junto al más reciente de creatividad táctica (Alarcón et al., 2017, Memmert, 2011b) son los más ampliamente mencionados en la actualidad, dentro de los estudios que relacionan la creatividad con el deporte (Domínguez, y Castillo, 2017).

Como ya se ha mencionado anteriormente, Memmert ha sido uno de los investigadores que han impulsado esta área de investigación, lo que queda constatado, puesto que, en 10 de los 39 documentos encontrados, él aparece entre los primeros autores. El origen alemán de las muestras de sus estudios se destaca en esta revisión sistemática, en la que 1,066 de 5,349 participantes, un $19.93 \%$, procedían de muestras de 


\section{García-Peñas et al.}

deportistas alemanes, siendo todos ellos pertenecientes a estudios de Memmert, salvo 56 participantes que eran de muestras alemanas y brasileñas pertenecientes al estudio de (Klatt et al., 2019).

Ahora bien, pese a que la presente revisión sistemática ha sido lo más abierta posible, dentro de unos criterios mínimos de selección, con el objetivo de abarcar el mayor número posible de estudios, debemos destacar que diversas publicaciones de las que se compone esta revisión sistemática han presentado una serie de limitaciones que se han considerado lo suficientemente destacables como para mencionarlas. Este tipo de matizaciones busca que futuras investigaciones cubran estas limitaciones y permitan a quienes se adentren en las investigaciones de creatividad y deporte alcanzar unos resultados más fiables y replicables.

Una de las principales limitaciones que han presentado 11 de los 39 estudios recopilados es que se hace mención a una muestra que practica deporte, o que lo ha practicado en los últimos años; obviando por completo mencionar el tipo de deporte que practican, la regularidad del mismo, el tiempo que llevaban practicándolo, o siquiera, si eran deportes de equipo o individuales.

La importancia de reflejar en estos datos anteriormente mencionados reside en lo que diversos autores comentan, como en el caso de Matthew et al. (2014), el cual nos habla sobre hallazgos importantes que relacionan el desarrollo de la creatividad con la práctica deportiva en entornos deportivos informales y no estructurados a lo largo del tiempo. Esto demostraría que, según el tipo de deporte practicado, la organización del mismo, así como el tiempo dedicado a éste, influiría en el desarrollo de la creatividad en los jóvenes. En la misma línea, Richard et al. (2017) remarcaron la importancia que tenían los años de experiencia en los deportes como efecto modulador principal en la creatividad de los propios atletas.

Otras de las limitaciones, es que en 5 de los 39 estudios no se hace mención alguna a la edad de la muestra, sin indicar si la muestra era menor o mayor de edad. Siguiendo en la línea de las limitaciones encontradas en las muestras de algunos de los estudios, podemos observar que, en 7 de los 39 estudios, del total de los
5,349 participantes que componen los 39 estudios, 897 de ellos no tenían una asignación en su condición de sexo. Por último, destacar que 4 de los 39 estudios compartían la característica de no aparecer especificado ni el sexo, ni la edad de la muestra, siendo esto una importante limitación a la hora de replicarlos. A pesar de que Baer y Kaufman (2008), referenciado en Martín-Brufau y Corbalán (2016), informan de la existencia de una extensa bibliografía científica sobre la diferencia en puntuaciones de creatividad entre hombres y mujeres a lo largo de los años; concluyen que no parece haber dichas diferencias significativas de sexo en relación a la creatividad general. Sin embargo, en este campo de la creatividad más específico, donde se podría englobar a los autores que la denominan como creatividad motriz o 'creatividad relacionada con el deporte', sería de gran utilidad para futuras investigaciones obtener datos sobre la diferencia en puntuaciones de creatividad motriz entre mujeres y hombres, con el fin de indagar cualquier posible diferencia significativa entre ambos sexos en relación a este tipo concreto de creatividad.

Para finalizar, se hace necesario indicar una limitación que se ha encontrado con frecuencia en los estudios que componen esta revisión sistemática, y es la manera en la que se ha evaluado la creatividad en algunos de los estudios. Tal y como mencionan Corbalán y Limiñana (2010), el uso de tests para evaluar la creatividad ha permitido generar medidas que puedan ser comparadas entre distintas personas. Es por ello, que en estos últimos años, la perspectiva psicométrica ha sido la más utilizada para la evaluación de la creatividad, a través de instrumentos estandarizados. Ya que, aunque un test puede resultar restrictivo, su esencia radica en poder predecir a partir de una mínima información, lo que puede y no puede ser plasmado en dicho test, y hacerlo o no de manera correcta. En esta línea, se ha de mencionar que, en 7 de los 39 estudios, se ha utilizado como método de evaluación de la creatividad el consenso de un 'criterio de expertos', en el cual ciertas personas bajo su criterio determinan qué movimientos o acciones son más o menos creativas a su parecer. A diferencia de la amplia serie de instrumentos estandarizados que existen en la actualidad para la evaluación de la creatividad, el criterio de expertos está sujeto a sesgos cognitivos de los propios evaluadores, pudiendo quizás distorsionar los resultados obtenidos en sus investigaciones y dificultando la replicabilidad de los mismos, a la hora 


\section{Creatividad y deporte}

de contrastar sus puntuaciones con los de otras investigaciones. No obstante, una de las razones por las que es posible que se optara por este tipo de evaluación en dichos estudios reside en lo que Méndez-Martínez (2019) indica acerca de que, aunque en la actualidad existen instrumentos que evalúan la creatividad motriz y sus dimensiones, dichos instrumentos no han sido diseñados y validados para la población menor de edad, puesto que las tareas propuestas suelen ser largas y complejas, y no permiten ser aplicados en una única sesión.

Si se tuviera que hacer una valoración rápida sobre cuál ha sido la cantidad de estudios que no poseían algunas o varias de las limitaciones anteriormente mencionadas, y sin entrar a hacer un cribado por criterios de calidad exhaustivo, la presente revisión tendría que haberse reducido a tan sólo 25 de los 39 estudios, habiéndose tenido que descartar un total de 14 estudios, lo que supone una cifra elevada.

\section{CONCLUSIONES}

Tras esta revisión sistemática, se puede concluir que de los 5,174 estudios que han sido revisados manualmente, para que cumplieran con el mínimo de los criterios de selección propuestos en el apartado de metodología del presente trabajo, se han obtenido un total de 39 documentos que versan sobre la temática abordada, superando dichos criterios tan sólo el $0.75 \%$ de la bibliografía revisada.

Se debe remarcar que 14 de los 39 estudios encontrados presentan ciertas limitaciones que dificultan su replicabilidad, del mismo modo que disminuyen en gran medida la calidad de sus resultados. Con la aplicación de los criterios de calidad extra para el cribado de los estudios seleccionados, se hubiera descartado más de un tercio de los estudios que componen la presente revisión sistemática.

En síntesis, cabe indicar que los hallazgos de este conjunto de estudios representativo resultan más bien dispersos, con un carácter marcadamente exploratorio. Más allá de los trabajos de Daniel Memmert, no puede decirse que se exista una línea investigación definitoria del campo de estudio, ni tampoco instrumentos de evaluación especializados que resulten ampliamente aceptados por la comunidad científica. El concepto de creatividad motriz (Domínguez-Iglesias et al., 2014; Méndez-Martínez, y Fernández-Río, 2019), o resulta prometedor, aunque, dada la dispersión de las propuestas de estudio, más bien cabe calificar el área como con un gran potencial de desarrollo.

Pese a las limitaciones de estos primeros estudios en la evaluación de la creatividad, no se debe obviar el enorme crecimiento exponencial que ha vivido esta área en el campo del deporte en los últimos 5 años, viéndose reflejada en las investigaciones realizadas por diferentes autores, buscando relacionar los niveles de creatividad con el éxito alcanzado por los atletas en sus respectivos deportes.

Esta línea de exploración presenta, pues, unas interesantes expectativas para tratar de responder a la cuestión de si puede hablarse de un dominio específico deportivo o motriz, dentro de los recursos de creatividad de las personas. Sin embargo, se ha de concluir que la investigación publicada hasta la fecha no ha hecho sino esbozar posibilidades y abrir líneas de trabajo, sin que pueda ofrecerse un conjunto de contenidos, estructura o relaciones para dicho constructo. A esa falta de definición contribuyen tanto las limitaciones metodológicas de una mayoría de estudios, como la falta de empleo de instrumentos objetivos de medida de la creatividad, así como también el moderado número global de trabajos en esta temática. No obstante, puede también observarse que, tanto por la tendencia de incrementos en la calidad y en la cantidad de las investigaciones, como por la incipiente acumulación de evidencias, puede hablarse del estudio de la creatividad deportiva como un área específica de la creatividad, considerándose ésta como una hipótesis suficientemente consolidada.

Es por ello que parece inevitable la necesidad de que emerja una estrategia específica para el estudio de la creatividad en el ámbito deportivo, máxime cuando el debate acerca de la especificidad o globalidad de la aptitud creativa sigue abierto (An, y Runco, 2016). En un amplio estudio con varios miles de sujetos evaluados acerca de su propia creatividad en 56 dominios discretos, Kaufman et al. (2009) concluyeron que el análisis proporcionó evidencia tanto de un factor general global como de siete áreas temáticas más específicas de desempeño creativo (artístico-verbal, artístico-visual, emprendedor, interpersonal, matemáticas-ciencia, desempeño y resolución de problemas), pero como se ve, éstas no 


\section{García-Peñas et al.}

incluían nada relativo a un posible dominio deportivo para la creatividad.

$\mathrm{Y}$, sin embargo, como tan acertadamente indicaron en su famoso artículo Kaufman, y Baer (2002), ¿Podría Steven Spielberg manejar a los Yankees? La respuesta más bien apunta a que no es nada fácil ser un genio creativo con éxito en más de un dominio. Eso podría deberse, sin duda, al cuantioso tiempo que lleva prepararse para un rendimiento de alto nivel en cualquier ámbito, incluido el deportivo. Pero también, quizás, al hecho de que los mecanismos cognitivos reales que subyacen al desempeño creativo son más específicos de cada dominio. Así, aunque algunos estudios sugieren la existencia de factores generales que influyen en la creatividad en todos los dominios, las evaluaciones reales del desempeño de la creatividad en diferentes dominios específicos generalmente encuentran poca evidencia de tales factores generales, y más bien encuentran lo contrario, con la probable excepción de g, el factor general de inteligencia. En cualquier caso, parece necesario profundizar en si la creatividad deportiva podría ser considerada como uno de esos dominios específicos, así como encuadrar el gran recurso que supone para el desarrollo del potencial humano. Para ello, se entiende relevante haber podido aportar la presente recopilación y revisión de la investigación acumulada a lo largo de las últimas décadas en torno a la creatividad en el contexto deportivo.

\section{APLICACIONES PRÁCTICAS}

Para un profesional del deporte, o de sus ciencias afines y especialmente de la psicología del deporte, el presente trabajo puede ser un recurso que posibilite su introducción en el conocimiento de las técnicas de creatividad en el contexto deportivo. Puede resultar indicativo de qué trabajos relacionados con la creatividad motriz, o con la creatividad táctica, pueden resultar más adecuados para su formación en la aplicación de una metodología creativa a su práctica profesional, o a su desarrollo como deportista. Esta investigación documental, asimismo puede facilitar el conocimiento de las relaciones entre la creatividad y otras variables de entrenamiento. Puede facilitar criterios para la selección de deportistas con presencia de características psicológicas de interés para la creatividad, y asimismo favorecer el conocimiento de en qué aspectos concretos de la práctica deportiva puede ésta incidir.

\section{REFERENCIAS}

1. Alarcón, F., Castillo, A., Ureña, N., Torre, E., y Cárdenas, D. (2017). Creatividad táctica y funciones ejecutivas en los deportes de interacción. SPORT TK-Rev. EuroAmericana de Ciencias del Deporte, 6(2), 147-152. https://doi.org/10.6018/300501

2. An, D., y Runco, M. A. (2016). General and domain-specific contributions to creative ideation and creative performance. Europe's journal of psychology, 12(4), 523-532. https://doi.org/10.5964/ejop.v12i4.1132

3. Aydin, E. (2019a). The Effect of Cultural Intelligence and Creative Thinking on the Practical Technical Capabilities of Trabzon University Physical Education Students. Asian Journal Of Education And Training, 5(2), 392-396. https://doi.org/10.20448/journal.522.2019.52. 392.396

4. Aydin, E. (2019b). The Effect of Spiritual Intelligence and Optimistic on Creativity in Team and Individual Sports Activities of Trabzon University Students. Asian Journal Of Education And Training, 5(3), 397-402. https://doi.org/10.20448/journal.522.2019.53. 397.402

5. Aoyagi, M. W., Portenga, S. T., Poczwardowski, A., Cohen, A. B. y Statler, T. (2012). Reflections and directions: The profession of sport psychology past, present, and future. Professional Psychology: Research and Practice, 43(1), 32-38. https://doi.org/10.1037/a0025676

6. Baer, J., y Kaufman, J. C. (2008). Gender differences in creativity. The Journal of Creative Behavior, 42(2), 75-105. https://doi.org/10.1002/j.21626057.2008.tb01289.x

7. Bernacka, R., Sawicki, B., Mazurek-Kusiak, A. y Hawlena, J. (2016). Conforming and nonconforming personality and stress coping styles in combat athletes. Journal Of Human 


\section{Creatividad y deporte}

Kinetics, 51(1), 225-233. https://doi.org/10.1515/hukin-2015-0186

8. Bohórquez-Gómez-Millán, M. y ChecaEsquiva, I. (2019). Desarrollo de competencias mediante ABP y evaluación con rúbricas en el trabajo en grupo en Educación Superior. REDU. Revista De Docencia Universitaria, 17(2), 197. https://doi.org/10.4995/redu.2019.9907

9. Bowers, M. T., Green, B. C., Hemme, F. y Chalip, L. (2014). Assessing the relationship between youth sport participation settings and creativity in adulthood. Creativity Research Journal, 26(3), 314-327. https://doi.org/10.1080/10400419.2014.9294 20

10. Burdzicka-Wołowik, J. y GóralRadziszewska, K. (2014). Selected Personality Traits of Women Training Combat Sports. Polish Journal Of Sport And Tourism, 21(1), 3-7. https://doi.org/10.2478/pjst-2014-0001

11. Cavallera, G., Boari, G., Labbrozzi, D. y Bello, E. (2011). Morningness-Eveningness Personality and Creative Thinking Among Young People Who Play Recreational Sport. Social Behavior And Personality: An International Journal, 39(4), 503-518. https://doi.org/10.2224/sbp.2011.39.4.503

12. Chacón-Araya, Y. y Moncada-Jiménez, J. (2006). Relación entre personalidad y creatividad en estudiantes de educación física. Revista Electrónica "Actualidades Investigativas en Educación", 6(1). ISSN: 1409-4703.

https://www.redalyc.org/articulo.oa?id=4476 0102

13. Colzato, L., Szapora, A., Pannekoek, J. y Hommel, B. (2013). The impact of physical exercise on convergent and divergent thinking. Frontiers In Human Neuroscience, 7 , 1-6. https://doi.org/10.3389/fnhum.2013.00824
14. Conesa-Ros, E., y Angosto, S. (2017). La expresión corporal y danza en la educación física de secundaria y bachillerato. Cuadernos de Psicología del Deporte, 17(2), 111-120. https://revistas.um.es/cpd/article/view/30196 1

15. Corbalán-Berná, J. y Limiñana-Gras, R.M. (2010). El genio en una botella. El test CREA, las preguntas y la creatividad. Introducción al monográfico "El test CREA, inteligencia creativa". Anales de Psicología/Annals of Psychology, 26(2), 197-205. https://revistas.um.es/analesps/article/view/1 08981

16. Cox, R. H. (2008). Psicología del deporte: conceptos y sus aplicaciones. Editorial Médica Panamericana.

17. Cropley, D. H., Cropley, A. J., Kaufman, J. C. y Runco, M. A. (Eds.). (2010). The dark side of creativity. Cambridge University Press. https://doi.org/10.1017/CBO9780511761225

18. Domínguez, C.L., y Castillo, E. (2017). Relación entre la danza libre-creativa y autoestima en la etapa de educación primaria. Cuadernos Psicol. del Deporte, 17(1), 73-80. https://revistas.um.es/cpd/article/view/29193

19. Domínguez-Iglesias, A.; Díaz-Pereira, M.P. y Martínez-Vidal, A. (2014). Estudio comparativo de los niveles de creatividad motriz en practicantes y no practicantes de expresión corporal. RETOS. Nuevas Tendencias en Educación Física, Deporte y Recreación, (26), 56-59. https://www.redalyc.org/articulo.oa?id=3457 32292010

20. Durand-Bush, N. y Salmela, J. H. (2002). The development and maintenance of expert athletic performance: Perceptions of world and Olympic champions. Journal of Applied Sport Psychology, 14(3), 154-171. https://doi.org/10.1080/10413200290103473

21. Furley, P. y Memmert, D. (2015). Creativity and working memory capacity in sports: 


\section{García-Peñas et al.}

working memory capacity is not a limiting factor in creative decision making amongst skilled performers.Frontiers In Psychol., 6, 17. https://doi.org/10.3389/fpsyg.2015.00115

22. Furley, P. y Memmert, D. (2018). Can creative role models prime creativity in soccer players? Psychology Of Sport And Exercise, 37, $1-9$. https://doi.org/10.1016/j.psychsport.2018.03. 007

23. Feist, G.J. y Runco, M.A. (1993). Trends in the creativity literature: An analysis of research in the Journal of Creative Behavior (1967-1989). Creativity Research Journal, 6 (3), 271-283 https://doi.org/ $10.1080 / 10400419309534483$

24. Garcés de los Fayos, E., Vives Benedicto, L. y Dosil, J. (2004). Nuevas aportaciones en Psicología del Deporte. Una mirada crítica sobre la última década de nuestra disciplina. Cuadernos Psic. Deporte, 4 (1 y 2), 7-16. https://revistas.um.es/cpd/article/view/93591

25. Guilford, J.P. (1967) The Nature of Human Intelligence. New York: McGraw Hill.

26. Hackfort, D. y Schinke, R. J. (Eds.). (2020). The Routledge International Encyclopedia of Sport and Exercise Psychology: Volume 1: Theoretical and Methodological Concepts. Routledge.

27. Hendry, D., Williams, A. y Hodges, N. (2018). Coach ratings of skills and their relations to practice, play and successful transitions from youth-elite to adultprofessional status in soccer. Journal of Sports Sciences, 36(17), 2009-2017. https://doi.org/10.1080/02640414.2018.1432 236

28. Herman-Tofler, L. y Tuckman, B. (1998). The effects of aerobic training on children's creativity, self-perception, and aerobic power. SPORT PSYCHIATRY, 7(4), 773-790. https://doi.org/10.1016/S10564993(18)30211-6
29. Hüttermann, S., Memmert, D. y Nerb, J. (2019). Individual differences in attentional capability are linked to creative decision making. Journal of Applied Social Psychology, 49(3), 159-167. https://doi.org/10.1111/jasp.12572

30. Karaca, N. y Aral, N. (2017). Adaptation of Thinking Creatively in Action and Movement Test for Turkish Children. Universidad Mehmet Akif Ersoy, Revista de la Facultad de Educación, 42, 240-253. https://doi.org/10.21764/efd.26968

31. Kaufman, J. C. y Baer, J. (2002). Could Steven Spielberg manage the Yankees? Creative thinking in different domains. Korean Journal of Thinking and Problem Solving, $\quad 12(2), \quad 5-14$. http://users.rider.edu/ baer/KaufmanBaerSpi elberg.pdf

32. Kaufman, J.C.; Cole, J.C. y Baer, J. (2009). The Construct of Creativity: Structural Model for Self-Reported Creativity Ratings. The Journal of Creative Behavior. 43(2). 119-134. https://doi.org/10.1002/j.2162-

6057.2009.tb01310.x

33. Klatt, S., Noël, B., Musculus, L., Werner, K., Laborde, S., Calábria-Lopes, M., Greco, P.J., Memmert, D. y Raab, M. (2019) Creative and Intuitive Decision-Making Processes: A Comparison of Brazilian and German Soccer Coaches and Players, Research Quarterly for Exercise and Sport, 9(4),651-665. https://doi.org/10.1080/02701367.2019.1642 994

34. Long, H., Plucker, J.A., Yu, Q., Ding, Y. y Kaufman, J.C. (2014). Research Productivity and Performance of Journals in the Creativity Sciences: A Bibliometric Analysis. Creativity Research Journal, 26 (3), 353-360. https://doi.org/10.1080/10400419.2014.9294 25.

35. Marín, A. A., Piñar, M., Camacho, P., Brox, V., Miranda-León, M., Suárez-Cadenas, E., 


\section{Creatividad y deporte}

Alarcón, F., y Cárdenas, D. (2012). Características de la personalidad de jóvenes jugadores de baloncesto (15-under). Cuadernos de Psicología del Deporte, 12(1), 135-138. https://doi.org/10.4321/S157884232012000100012

36. Martín-Brufau, R. y Corbalán, J. (2016). Creativity and Psychopathology: Sex Matters, Creativity Research Journal, 28(2), 222-228. https://doi.org/10.1080/10400419.2016.1165 531

37. Martínez-Vidal, A. y Díaz-Pereira, P. (2008). Creatividad y deporte: consideraciones teóricas e investigaciones breves. Wanceulen.

38. Matthew T. Bowers, B. Christine Green, Florian Hemme y Laurence Chalip (2014) Assessing the Relationship Between Youth Sport Participation Settings and Creativity in Adulthood, Creativity Research Journal, 26(3), 314-327. https://doi.org/10.1080/10400419.2014.9294 20

39. Meier, J. (2014). Creativity, Innovation y Change in the World of Sports. ( 9 de Octubre de 2016). Championship Thinking Coach. http://www.championshipthinkingcoach.com /expertise/innovation-a-change.html

40. Memmert, D. (2006). Developing creative thinking in a gifted sport enrichment program and the crucial role of attention processes. High Ability Studies, 17(1), 101115.

https://doi.org/10.1080/13598130600947176

41. Memmert, D. (2007). Can Creativity Be Improved by an Attention-Broadening Training Program? An Exploratory Study Focusing on Team Sports. Creativity Research Journal, 19(2-3), 281-291. https://doi.org/10.1080/10400410701397420

42. Memmert, D. (2010). Testing of tactical performance in youth elite soccer. Journal of Sports Science And Medicine, 9, 1-7.
https://www.ncbi.nlm.nih.gov/pmc/articles/P MC3761738/

43. Memmert, D. (2011a). Creativity, expertise, and attention: Exploring their development and their relationships. Journal of Sports Sciences, 29(1), 93-102. https://doi.org/10.1080/02640414.2010.5280 14

44. Memmert, D. (2011b). Sports and Creativity. In: Runco MA, and Pritzker SR (Eds.) Encyclopedia of Creativity, (2 ${ }^{\circ}$ ed., Vol. 2, pp. 373-378). San Diego: Academic Press.

45. Memmert, D. (2015). Teaching Tactical Creativity in Sport: Research and Practice. Routledge.

https://doi.org/10.4324/9781315797618

46. Memmert, D., Baker, J. y Bertsch, C. (2010). Play and practice in the development of sportspecific creativity in team ball sports. High Ability Studies, 21(1), 3-18. https://doi.org/10.1080/13598139.2010.4880 83

47. Memmert, D. y Perl, J. (2009). Game creativity analysis using neural networks. Journal Of Sports Sciences, 27(2), 139-149.

https://doi.org/10.1080/02640410802442007

48. Memmert, D. y Roth, K. (2007). The effects of non-specific and specific concepts on tactical creativity in team ball sports. Journal Of Sports Sciences, 25(12), 1423-1432. https://doi.org/10.1080/02640410601129755

49. Méndez-Martínez, E. y Fernández-Río, J. (2019). Diseño y validación de un instrumento de medición de la creatividad motriz en adolescentes. Revista Internacional De Medicina Y Ciencias De La Actividad Física $Y$ Del Deporte, 19(75), 535-550. https://doi.org/10.15366/rimcafd2019.75.010

50. Meric, E. (2014). The analysis of the thinking styles and creativity of the sports students studying in the different fields of 


\section{García-Peñas et al.}

university. Educational Research And

Reviews, 9(20), 866-871. https://doi.org/10.5897/err2014.1783

51. Noorafshan, L., Fazli, F. y Tavousisimakani, R. (2016). MSCwellbeing of the athletes. International Journal Of Pharmaceutical Research y Allied Sciences, 5(3), 448-457. ISSN: 2277-3657.

52. Orth, D., McDonic, L., Ashbrook, C. y Van der Kamp, J. (2019). Efficient search under constraints and not working memory resources supports creative action emergence in a convergent motor task. Human Movement Science, 67, 102505. https://doi.org/10.1016/j.humov.2019.102505

53. Ortín-Montero, F.J., Moya-Faz, F., y Garcés de los Fayos, E.J. (2013). Sport Psychology: Some new proposals being developed. Anales de Psicología, 29(3), 633-636. https://doi.org/10.6018/analesps.29.3.175741

54. Pepe, O. (2018). The Impact of The Creativity Levels of The Students Who Study at The School of Physical Education and Sport on Their Critical Thinking Dispositions. World Journal Of Education, 8(5), 185-191. https://doi.org/10.5430/wje.v8n5p185

55. Pérez Ramírez, M.C. (2002). Caracterización del entrenador de alto rendimiento deportivo. Cuadernos Psicología Deporte, 2(1), 15-37. https://revistas.um.es/cpd/article/view/10074 1

56. Predoiu, R., Mitrache, G. y Predoiu, A. (2016). The effects of induced emotions on working memory, creativity, repetition speed and precision: a pilot study. Physical Education, Sport And Kinetotherapy Journal, 12(2), 44-51. http://discobolulunefs.ro/Reviste/2016/DISC OBOLUL_2_44_2016_FULL_v2.pdf\#page= 43

57. Puig, N. y Heinemann, K. (1991). El deporte en la perspectiva del año 2000. Papers: revista de sociologia, 38, 123-141.
58. Putwain, D. W., Kearsley, R. y Symes, W. (2012). Do creativity self-beliefs predict literacy achievement and motivation? Learning and Individual Differences, $\quad 22(3), \quad 370-374$. https://doi.org/10.1016/j.lindif.2011.12.001

59. Rasmussen, L. y Østergaard, L. (2016). The Creative Soccer Platform: New Strategies for Stimulating Creativity in Organized Youth Soccer Practice. Journal of Physical Education, Recreation y Dance, 87(7), 9-19. https://doi.org/10.1080/07303084.2016.1202 799

60. Richard, V., Abdulla, A. y Runco, M. (2017). Influence of Skill Level, Experience, Hours of Training, and Other Sport Participation on the Creativity of Elite Athletes. Journal of Genius And Eminence, 2(1), 65-76. http://doi.org/10.18536/jge.2017.04.02.01.07

61. Richard, V. y Runco, M. A. (2020). Creativity: The Emergence of a New Dimension of Sport Expertise. In Tenenbaum, G., y Eklund, R. C. (Eds.), Handbook of sport psychology. Hoboken. NJ: Wiley.

62. Runco, M. A. (2004). Creativity. Annual Review of Psychology, 55, 657-687. https://doi.org/10.1146/annurev.psych.55.090 902.141502

63. Runco, M.A. (2014). Creativity: Theories and themes: Research, development, and practice. San Diego, CA: Elsevier Academic Press.

64. Runco, M. A. y Pritzker, S. R. (Eds.). (2011). Encyclopedia of creativity. San Diego. Academic Press.

65. Rutkowska, K. y Gierczuk, D. (2012). Selected Cognitive And Emotional Resources Of Untrained Youth And Young Wrestlers. Polish Journal Of Sport And Tourism, 19(3), 190-195. https://doi.org/10.2478/v10197012-0018-x 


\section{Creatividad y deporte}

66. Said-Metwaly, S., Noortgate, W. y Kyndt, E. (2017). Approaches to Measuring Creativity: A Systematic Literature Review. Creativity. Theories - Research - Applications, 4(2), 238275. https://doi.org/10.1515/ctra-2017-0013

67. Santos, S., Memmert, D., Sampaio, J. y Leite, N. (2016). The Spawns of Creative Behavior in Team Sports: A Creativity Developmental Framework. Frontiers Psychology, 7. 1282. https://doi.org/10.3389/fpsyg.2016.01282

68. Santos, S., Jiménez, S., Sampaio, J. y Leite, N. (2017). Effects of the Skills4Genius sportsbased training program in creative behavior. PLOS ONE, 12(2), e0172520. https://doi.org/10.1371/journal.pone.0172520

69. Simonton, D.K. (2012). Creative Genius as a Personality Phenomenon. Social and Personality Psychology Compass, 6 (9), 691$706 . \quad$ https://doi.org/10.1111/j.17519004.2012.00455.x

70. Taylor, J. E. y Wilson, G. E. (2005). Applying sport psychology: Four perspectives. Human Kinetics.

71. Tedesco da Costa, Trevisan P.R., y Schwartz, G.M. (2018). Produção do conhecimento: uma revisão sistemática sobre criatividade motora. Actualidades Investigativas en Educación, $\quad 18(1), \quad 67-98$. https://doi.org/10.15517/aie.v18i1.31320

72. Thomson, P. y Victoria-Jaque, S. (2016). Visiting the Muses: Creativity, Coping, and PTSD in Talented Dancer and Athletes. American Journal Of Play, 8(3), 363-378. https://files.eric.ed.gov/fulltext/EJ1108799.p df

73. Tohănean, I. (2016). Study on the level of creativity for the female handball players (Seniors). Series IX: Sciences Of Human Kinetics, 9(2), 69-74.

74. Top, E. y Akil, M. (2018). Effects of the Sports on the Personality Traits and the
Domains of Creativity. World Journal of Education, $\quad 8(3), \quad 56-64$. https://doi.org/10.5430/wje.v8n3p56

75. Torrance, E.P. y Myers, R.E. (1976). La enseñanza creativa. Madrid. Santillana.

76. Torrebadella Flix, X. (2012). El deporte contra la educación física. Revista Movimiento Humano, 4, 73-98.

77. Urrútia, G. y Bonfill, X. (2010). Declaración PRISMA: una propuesta para mejorar la publicación de revisiones sistemáticas y metaanálisis. Medicina Clín 135(11), 507-511 https://doi.org/10.1016/j.medcli.2010.01.015

78. Veraksa, A. y Gorovaya, A. (2012). Imagery training efficacy among novice soccer players. Procedia-Soc.Beh.Sci., 33, 338-342 https://doi.org/10.1016/j.sbspro.2012.01.139

79. Vestberg, T., Gustafson, R., Maurex, L., Ingvar, M. y Petrovic, P. (2012). Executive Functions Predict the Success of Top-Soccer Players. Plos ONE, 7(4), e34731. https://doi.org/10.1371/journal.pone.0034731

80. Wen-Yu, C. y Jhao-You, H. (2017). A Study Of Creative Teaching And Technological Creativity Using Hierarchical Linear Models. The International Journal Of Organizational Innovation, 9(1), 60-73.

81. Williams, R., Runco, M.A. y Berlow, E. (2016). Mapping the Themes, Impact, and Cohesion of Creativity Research over the Last 25 Years. Creativity Research Journal, 28 (4), 385-394. https://doi.org/10.1080/1040 0419.2016.1230358.

82. Zoljanahi, E., Mohammadi-nezhade, M., Sadeghpour Gilde, B. y Taheri, H. (2018). Investigating Sport Attributional Style, Sport Self-Efficacy, and Creativity in Elite Team Athletes. Iranian Journal Of Psychiatry And Behavioral Sciences, 12(3), e7860. https://doi.org/10.5812/ijpbs.7860 